\title{
Os tipos estáveis e multiplicidades de germes quase homogêneos de $\mathbb{C}^{n}$ em $\mathbb{C}^{n}$
}

\author{
Aldicio José Miranda
}


Data de depósito: 29-10-2004

Assinatura:

\title{
Os tipos estáveis e multiplicidades de germes quase homogêneos de $\mathbb{C}^{n}$ em $\mathbb{C}^{n}$
}

\author{
Aldicio José Miranda
}

Orientador: Prof. Dr. Marcelo José Saia

Dissertação apresentada ao Instituto de Ciências Matemáticas e de Computação - ICMC-USP, como parte dos requisitos para obtenção do título de Mestre em Matemática.

USP - São Carlos

Outubro/2004 
Aos meus pais

Jovelina e

Geraldo (in memorian) 


\section{Agradecimentos}

À Deus, por tudo que me deste.

À minha família, que sempre torce por mim. Ao meu Pai Geraldo, que foi para mim um exemplo de vida, meu porto seguro e que sempre me apoiou, que saudades! que o senhor descanse em Paz. À Jovelina, minha mãe querida, obrigado pela presença em minha vida, por seus ensinamentos, por suas orações, que sempre pede a Deus por indicar-me o melhor caminho. Agradeço a vocês por tudo que hoje sou.

Ao Prof. Marcelo Saia, por tudo que me proporcionou com sua forte atenção nos ensinamentos. Obrigado por me orientar neste trabalho, sua dedicação e paciência foram fundamentais para a conclusão deste.

Ao Prof. Vitor Hugo e a Eliris, pela força que me deram, obrigado por terem dedicados boa parte do tempo de vocês em esclarecer minhas questões.

À todos os professores que de alguma forma contribuíram para o meu aprendizado.

Aos amigos do futebol: Saia, João Carlos, Lizandro, Marcos, Zé Paulo, Cássio, João Paulo, Ricardo, Raimundo, Márcio, Helton, Christian, Olivâine, Rodrigo e Sandro pelos momentos de descontração e alegria.

À todos que direta ou indiretamente contribuíram para a realização deste trabalho.

À CAPES pelo apoio financeiro. 


\section{Resumo}

A determinação dos invariantes numéricos associados a germes de aplicações diferenciáveis é uma ferramenta muito útil no estudo de problemas de equisingularidade em famílias. Em geral, estes invariantes são obtidos algebricamente através de esquemas $r$-dimensionais, que surgem nos tipos estáveis de uma perturbação estável do germe.

Neste trabalho é feito um estudo sobre estes invariantes nos tipos estáveis de germes de aplicações holomorfas $f:\left(\mathbb{C}^{n}, 0\right) \rightarrow\left(\mathbb{C}^{n}, 0\right)$ finitamente determinados de coposto 1 . Inicialmente é feita uma caracterização completa de todos os tipos estáveis, bem como de sua geometria. Como aplicações são estudados os invariantes no discriminante de germes quase homogêneos. São descritas fórmulas para os invariantes 0 -estáveis de germes de $\left(\mathbb{C}^{n}, 0\right) \rightarrow\left(\mathbb{C}^{n}, 0\right)$. Estes resultados são aplicados para o cálculo das multiplicidades polares do discriminante de germes quase homogêneos de $\left(\mathbb{C}^{3}, 0\right) \rightarrow\left(\mathbb{C}^{3}, 0\right)$. 


\section{Abstract}

The determination of the numerical invariants associated to map germs is a helpful tool in the study of problems of equisingularity in families. In general, these invariants are given as zero schemes, that appear in the stable types of a stable perturbation of the germ.

In this work we study the invariants in the stable types of corank one finitely determined holomorphic map germs $f:\left(\mathbb{C}^{n}, 0\right) \rightarrow\left(\mathbb{C}^{n}, 0\right)$. First we completely characterize all stable types and study their geometry. As applications are studied the invariants in the discriminant of weighted homogeneous germs. Formulas are described for the 0 -stable invariants of map germs of $\left(\mathbb{C}^{n}, 0\right) \rightarrow\left(\mathbb{C}^{n}, 0\right)$ and these results are applied to compute the polar multiplicities of the discriminant of weighted homogeneous germes of $\left(\mathbb{C}^{3}, 0\right) \rightarrow\left(\mathbb{C}^{3}, 0\right)$. 


\section{Sumário}

Introdução

1 Definições básicas e resultados gerais para caracterização de estabilidade 6

1.1 Germes de tipo finito e tipos estáveis . . . . . . . . . . . . . . 6

1.2 Teorema de Mather para estabilidade . . . . . . . . . . . . . . . . 8

1.3 Comprimento e multiplicidades de ideais . . . . . . . . . . . . . . 9

1.4 Aplicação finita e grau . . . . . . . . . . . . . . . . . . . . . 10

2 Caracterização dos tipos estáveis em germes finitamente determinados 12

2.1 Os tipos estáveis em $\mathscr{O}(n, n), r$-esquemas $\ldots \ldots \ldots \ldots \ldots \ldots \ldots \ldots$

2.2 Algoritmo para os tipos estáveis na fonte . . . . . . . . . . . . . . 24

3 Multiplicidades de 0-esquemas de germes quase homogêneos em $\mathscr{O}(n, n) \quad 31$

4 Multiplicidades polares do discriminante em germes quase homogêneos em $\mathscr{O}(3,3) \mathbf{3 7}$

4.1 Multiplicidades polares no discriminante . . . . . . . . . . . . . 37

4.2 Fórmulas para multiplicidades polares em $\Delta(f) \ldots \ldots \ldots \ldots \ldots$

$4.3 \quad$ Multiplicidades polares de $f\left(\Sigma^{1,1}(f)\right) \ldots \ldots \ldots \ldots \ldots \ldots$

4.4 Multiplicidades polares de $f\left(D_{1}^{2}(f / \Sigma(f))\right) \ldots \ldots \ldots \ldots \ldots$

5 Apêndice: Demonstração do Lema 2.1 44

$\begin{array}{ll}\text { Bibliografia } & 51\end{array}$ 


\section{Introdução}

A determinação dos invariantes numéricos associados a germes de aplicações diferenciáveis é uma ferramenta muito útil no estudo de problemas de equisingularidade em famílias. Em geral, estes invariantes são dados como esquemas zero dimensionais, que surgem nos tipos estáveis de uma perturbação estável do germe.

Como exemplo, citamos um resultado de Lê e Ramanujam de 1976, onde é mostrado que a constância de um único invariante numérico, o número de Milnor denotado por $\mu$, em uma família a 1-parâmetro de hipersuperfícies com singularidade isolada implica na trivialidade topológica da família. Mais precisamente, o número de Milnor é um invariante topológico no se-

guinte sentido: se $\{X(t)\}$ é uma família de hipersuperficies $n$-dimensionais em $\mathbb{C}^{n+1}$ com $n \neq 2$ com singularidade isolada em 0 , tal que $\mu(X(t))$ é independente de t, então o tipo topológico local da hipersuperfície em 0 necessariamente é constante.

Observamos que no contexto deste trabalho, o número de Milnor é o invariante 0-estável associado ao conjunto $\Sigma(f)$. Generalizações deste resultado clássico para interseções completas com singularidade isolada (I.C.I.S) usam a noção de regularidade de Whitney e as multiplicidades polares como invariantes numéricos [veja [12]].

O método para se determinar estes invariantes é considerar uma deformação estável do germe e contar as singularidades que aparecem nesta. Algebricamente podemos contar estas singularidades através da determinação das multiplicidades dos $r$-esquemas que ocorrem nos tipos estáveis. Podemos ver nos exemplos abaixo o cálculo dos invariantes 0-estáveis que surgem no discriminante.

1. Considere a aplicação

$$
\begin{gathered}
f: \mathbb{C}^{2} \rightarrow \mathbb{C}^{2} \\
(x, z) \mapsto\left(x, z^{4}+x z\right)
\end{gathered}
$$


e sua perturbação estável

$$
f_{\varepsilon}(x, z)=\left(x, z^{4}+x z+\varepsilon z^{2}\right)
$$

O conjunto singular de $f$ é uma curva suave dada por:

$$
\Sigma(f)=\left\{(x, z) \in \mathbb{C}^{2} / 4 z^{3}+x=0\right\}
$$

e o conjunto singular de $f_{\varepsilon}$ também é uma curva suave dada por:

$$
\Sigma\left(f_{\varepsilon}\right)=\left\{(x, z) \in \mathbb{C}^{2} / 4 z^{3}+x+2 \varepsilon z=0\right\} .
$$

O discriminante $\Delta(f)=f(\Sigma(f))$ de $f$ é uma curva com um ponto singular na origem, mas $\Delta\left(f_{\varepsilon}\right)=f_{\varepsilon}\left(\Sigma\left(f_{\varepsilon}\right)\right)$ é uma curva com duas cúspides e um ponto duplo que são chamados de $\left(\right.$ pontos $\left.-A_{2}\right)$ e $\left(\right.$ pontos $\left.-A_{1,1}\right)$ respectivamente.

Parametrizações para o discriminante de $f$ e de $f_{\varepsilon}$ são respectivamente dadas por:

$$
\begin{gathered}
t \mapsto\left(-4 t^{3},-3 t^{4}\right) \\
\mathrm{e} \\
t \mapsto\left(-4 t^{3}-2 \varepsilon t,-3 t^{4}-\varepsilon t^{2}\right)
\end{gathered}
$$
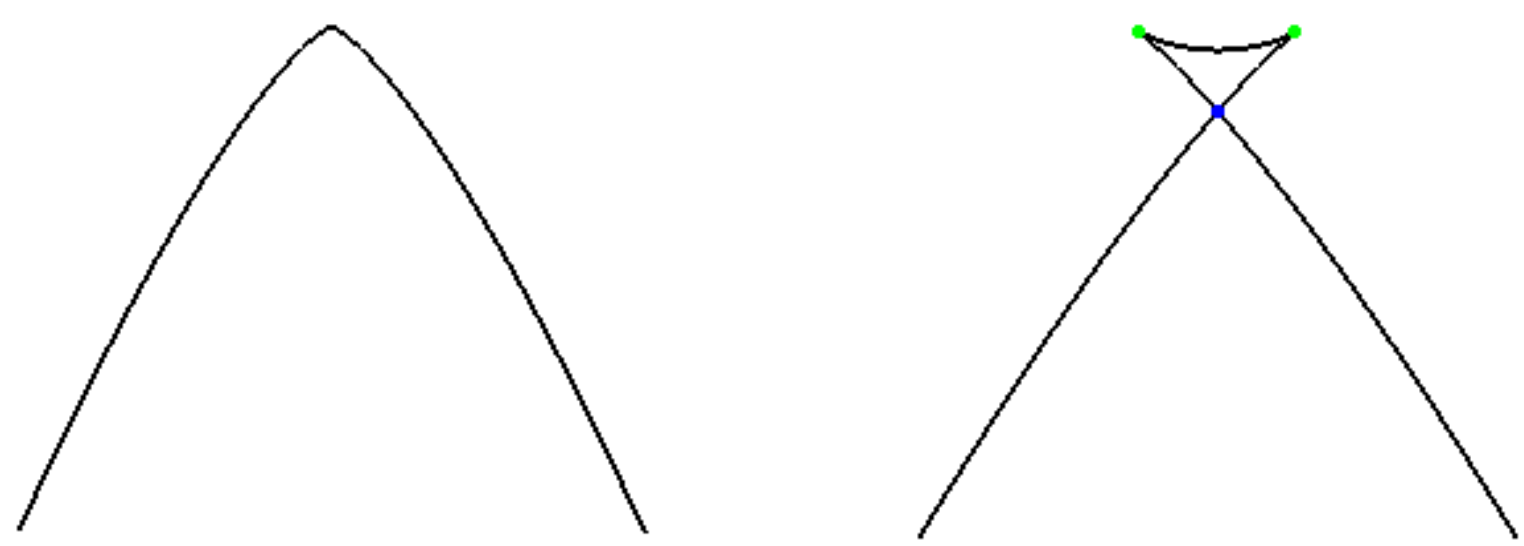

Figura 1: Discriminante de $f$ e $f_{\varepsilon}$ - o rabo de andorinha (parte real)

Observamos que o número de cúspides e de pontos duplos independe da perturbação, com a condição que a perturbação é uma aplicação estável. No exemplo acima, os zero-esquemas (tipos estáveis de dimensão zero) que aparecem são as cúspides e o ponto duplo (pontos nas cores verde 
e azul respectivamente, que aparecem na Figura 1.), como temos duas cúspides (pontos $\left.-A_{2}\right)$ sua multiplicidade é 2 e como temos um ponto duplo (pontos $-A_{1,1}$ ) sua multiplicidade é 1 .

No caso de germes finitamente determinados $f:\left(\mathbb{C}^{2}, 0\right) \rightarrow\left(\mathbb{C}^{2}, 0\right)$, T. Gaffney e D. Mond [7] mostram uma fórmula geral para determinar ambos os casos, ou seja, o número de cúspides e o número de pontos duplos de um germe de aplicação finitamente determinado.

2. Seja a aplicação

$$
\begin{gathered}
f: \mathbb{C}^{3} \rightarrow \mathbb{C}^{3} \\
(x, y, z) \mapsto\left(x, y, z^{5}+x z^{2}+y z\right)
\end{gathered}
$$

uma perturbação estável $f_{\varepsilon}$ de $f$ pode ser dada por:

$$
f_{\varepsilon}(x, y, z)=\left(x, y, z^{5}+x z^{2}+y z+\varepsilon z^{3}\right)
$$

Aqui o conjunto singular de $f$ é uma superfície suave em $\mathbb{C}^{3}$ dada por:

$$
\Sigma(f)=\left\{(x, y, z) \in \mathbb{C}^{3} / 5 z^{4}+2 x z+y=0\right\}
$$

e o conjunto singular de $f_{\varepsilon}$ também é uma superfície suave em $\mathbb{C}^{3}$ dada por:

$$
\Sigma\left(f_{\varepsilon}\right)=\left\{(x, y, z) \in \mathbb{C}^{3} / 5 z^{4}+2 x z+y+3 \varepsilon z^{2}=0\right\} .
$$

Parametrizações para o discriminante de $f$ e $f_{\varepsilon}$ são dadas respectivamente por:

$$
\begin{gathered}
(s, t) \mapsto\left(s,-5 t^{4}-2 s t,-4 t^{5}-s t^{2}\right) \\
\mathrm{e} \\
(s, t) \mapsto\left(s,-5 t^{4}-2 s t-3 \varepsilon t^{2},-4 t^{5}-s t^{2}-2 \varepsilon t^{3}\right)
\end{gathered}
$$

Os zero-esquemas de $\Delta\left(f_{\varepsilon}\right)$ são os 2 rabos de andorinha que são chamados de (pontos $-A_{3}$, pontos na cor verde da Figura 2), e os dois pontos onde o eixo cuspidal cruza transversalmente a folha suave, denominados (pontos $-A_{2,1}$, pontos na cor amarela da Figura 2 ). Logo a multiplicidade de cada um destes tipos 0-estáveis é 2. O ponto triplo não ocorre neste exemplo.

A Figura 2 apresenta os tipos $r$-estáveis, com $0 \leq r \leq 2$ na fonte e na meta. A superfície 

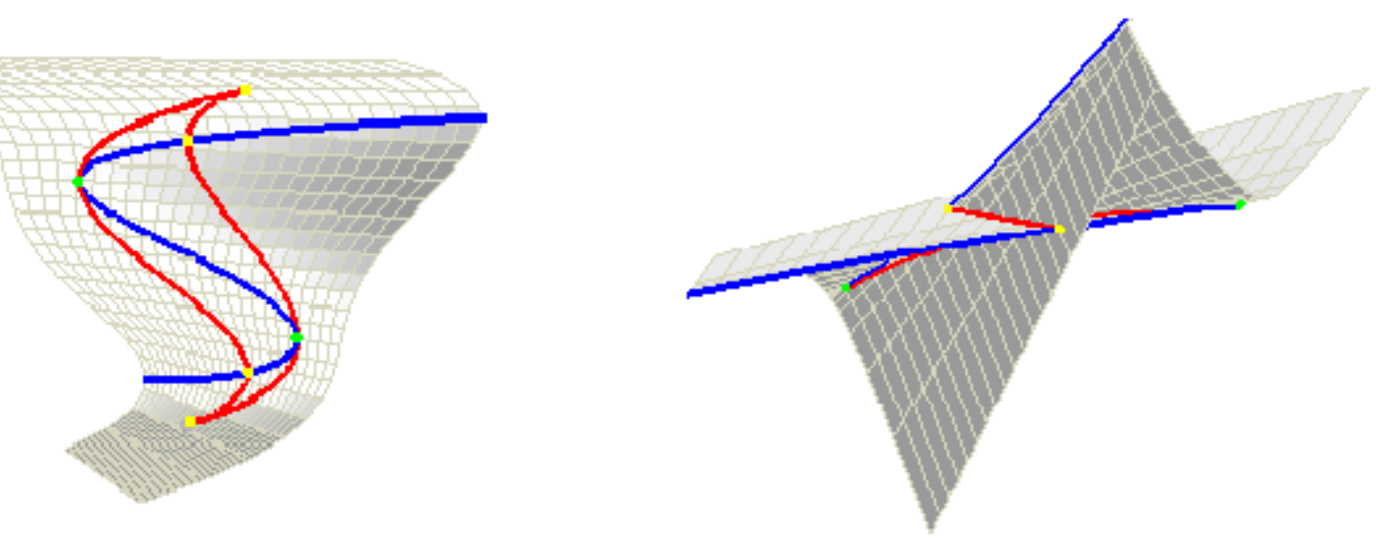

Figura 2: Conjunto singular e discriminante de $f_{\varepsilon}$ (parte real)

esquerda da Figura 2 é o conjunto singular de $f_{\varepsilon}$ (denotado por $\Sigma\left(f_{\varepsilon}\right)$ ) é um tipo estável de dimensão 2 na fonte. Os pontos nas cores verde e amarela contidos em $\Sigma\left(f_{\varepsilon}\right)$ são os tipos estáveis zero dimensionais na fonte. As curvas nas cores azul e vermelha contidas em $\Sigma\left(f_{\varepsilon}\right)$ são os tipos estáveis de dimensão 1, chamadas respectivamente de curva cuspidal e curva de pontos duplos na fonte. A superfície direita da Figura 2 é o discriminante de $f_{\varepsilon}$ (denotado por $\left.\Delta\left(f_{\varepsilon}\right)\right)$ é tipo estável de dimensão 2. Os pontos nas cores verde e amarela contidos em $\Delta\left(f_{\varepsilon}\right)$ são os tipos estáveis zero dimensionais na meta que mencionamos no parágrafo anterior. As curvas nas cores azul e vermelha contidas em $\Delta\left(f_{\varepsilon}\right)$ são os tipos estáveis de dimensão 1 na meta, chamadas respectivamente de curva cuspidal e curva de pontos duplos. Os tipos estáveis na meta são obtidos pela imagem de cada um dos tipos estáveis contidos em $\Sigma\left(f_{\varepsilon}\right)$ por $f_{\varepsilon}$, mantendo-se as dimensões.

Estamos considerando aqui o problema análogo para germes de aplicações $f: \mathbb{C}^{n} \rightarrow \mathbb{C}^{n}$, ou seja, para uma perturbação estável de $f$, perguntamos quais os tipos de singularidades que existem em $\Delta\left(f_{\varepsilon}\right)$, a mesma questão é feita para o conjunto singular $\Sigma\left(f_{\varepsilon}\right)$ e para o conjunto $X(f):=\overline{\left(f^{-1}(\Delta(f))-\Sigma(f)\right)}$. Os tipos estáveis são chamados de r-esquemas e o número de cada tipo são as multiplicidades. Também é estudada neste trabalho a questão de determinarmos fórmulas para o cálculo do número de singularidades, mas somente consideramos esta questão para os tipos estáveis do discriminante de germes quase homogêneos.

O principal objetivo deste trabalho é a caracterização dos tipos estáveis em deformações estáveis de germes finitamente determinados, o que foi feito no capítulo 2. Neste capítulo é feita uma descrição geral de todos os tipos estáveis, ou seja os $r$-esquemas, com $0 \leq r \leq n-1$, 
para os germes finitamente determinados de $\mathbb{C}^{n}$ em $\mathbb{C}^{n}$ de coposto 1 . No final do capítulo 2 é apresentado um algoritmo que calcula os ideias que define os tipos estáveis na fonte.

No capítulo três são obtidas fórmulas para determinar os invariantes 0-estáveis do discriminante de germes de coposto 1 quase homogêneos finitamente determinados $f:\left(\mathbb{C}^{n}, 0\right) \rightarrow\left(\mathbb{C}^{n}, 0\right)$.

No capítulo quatro são determinadas fórmulas para o cálculo das multiplicidades polares associadas aos $r$-esquemas, com $0 \leq r \leq 2$, do discriminante de germes de aplicações quase homogêneos de $\left(\mathbb{C}^{3}, 0\right)$ em $\left(\mathbb{C}^{3}, 0\right)$.

No apêndice é feita a demonstração do Lema 2.1, onde é descrito como são obtidos os ideais de definição dos $r$-esquemas de pontos múltiplos. Observamos que esta demonstração é muito técnica e sua introdução no corpo do texto iria atrapalhar sua leitura. Por isto foi colocada no apêndice. 


\section{Capítulo}

1

\section{Definições básicas e resultados gerais para}

\section{caracterização de estabilidade}

\subsection{Germes de tipo finito e tipos estáveis}

Definição 1.1. Sejam $f: M \rightarrow \mathbb{C}^{p}$ e $g: N \rightarrow \mathbb{C}^{p}$ duas aplicações holomorfas definidas em vizinhanças $M$ e $N$ de um ponto $p \in \mathbb{C}^{n}$, $f$ é equivalente a $g(f \sim g)$ se existir uma vizinhança $U$ de $p$, com $U \subset M \cap N$ tal que as restrições $f_{\mid U}$ e $g_{\mid U}$ coincidem.

Estas classes de equivalência são chamadas de germes de aplicações no ponto $p$.

Definição 1.2. Se $S \subset \mathbb{C}^{n}$ é tal que $f(x)=z$ para todo $x \in S$ então $f$ é chamado de multigerme no conjunto $S$ e que será denotado por $f:\left(\mathbb{C}^{n}, S\right) \rightarrow\left(\mathbb{C}^{p}, z\right)$.

Denota-se por $\mathscr{O}(n, p)$ o conjunto dos germes de aplicações holomorfas $f:\left(\mathbb{C}^{n}, 0\right) \rightarrow\left(\mathbb{C}^{p}, 0\right)$ e por $\mathscr{O}_{e}(n, p)$ o conjunto dos germes de aplicações holomorfas $f:\left(\mathbb{C}^{n}, 0\right) \rightarrow \mathbb{C}^{p}$. O anel local $\mathscr{O}_{e}(n, 1)$ é denotado por $\mathscr{O}_{n}$ e $m_{n}=\left\{f \in \mathscr{O}_{n} \mid f(0)=0\right\}$ denota seu ideal maximal.

Denota-se por $\mathscr{R}$ (ação à direita) o grupo de difeomorfismos da fonte $\left(\mathbb{C}^{n}, 0\right)$ e por $\mathscr{L}$ (ação $\grave{a}$ esquerda) o grupo de difeomorfismos da meta $\left(\mathbb{C}^{p}, 0\right)$. Da ação do produto $(\mathscr{R} \times \mathscr{L}):=\mathscr{A}$ resulta a $\mathscr{A}$ - equivalência de germes de aplicações: $f, g \in \mathscr{O}(n, p)$ são $\mathscr{A}$ - equivalentes se são equivalentes por mudanças holomorfas na fonte e na meta.

Definição 1.3. Um desdobramento a s-parâmetros do germe $f$ é um germe $F \in \mathscr{O}(s+n, s+p)$

$$
\begin{gathered}
F:\left(\mathbb{C}^{s} \times \mathbb{C}^{n},(0,0)\right) \rightarrow\left(\mathbb{C}^{s} \times \mathbb{C}^{p},(0,0)\right) \\
(u, x) \mapsto\left(u, f_{u}(x)\right)
\end{gathered}
$$


tal que $f_{0}(x)=f(x)$. O germe $f_{u}(x)$ é chamado uma deformação de $f$.

\section{Definição 1.4.}

i. F é um desdobramento trivial de $f$, se existem um desdobramento $R$ a s-parâmetros da identidade em $\mathbb{C}^{n}$ e um desdobramento L a s-parâmetros da identidade em $\mathbb{C}^{p}$ tal que

$$
L \circ F \circ R^{-1}=\left(I_{d}, f\right)
$$

ii. Dois desdobramentos à s-parâmetros $F_{1}$ e $F_{2}$ de f são isomorfos se $F_{2}=L \circ F_{1} \circ R^{-1}$ com $L$ e R desdobramentos da identidade.

iii. Um desdobramento à s-parâmetros $F_{2}$ é induzido de um desdobramento à t-parâmetros $F_{1}$ se existe uma aplicação $h:\left(\mathbb{C}^{s}, 0\right) \rightarrow\left(\mathbb{C}^{t}, 0\right)$ tal que $F_{2}=h^{*} F_{1}=\left(I_{d}, f_{u}(h(u), x)\right)$

Definição 1.5. F é um desdobramento versal de $f$ se todo desdobramento de $f$ é isomorfo a um desdobramento induzido de F.

Duas aplicações $f, g:\left(\mathbb{C}^{n}, a\right) \rightarrow\left(\mathbb{C}^{p}, b\right)$ tem o mesmo $k$-jato em $a$ se com respeito a algum sistema de coordenadas, todas as derivadas de $f$ e $g$ em $x=a$ coincidem até a ordem $k$.

Um dado germe de aplicação $f \in \mathscr{O}(n, p)$ é $k-\mathscr{G}$ - determinado $(\mathscr{G}=\mathscr{R}, \mathscr{L}, \mathscr{A}$ ou $\mathscr{K})$ se para todo $g \in \mathscr{O}(n, p)$ tal que os $k$-jatos $j^{k} g(0)$ e $j^{k} f(0)$ são iguais, $g$ é $\mathscr{G}$-equivalente a $f ; f$ é $\mathscr{G}-$ determinado se é $k-\mathscr{G}$ - determinado para algum $k<\infty$. Reservaremos a frase finitamente determinado para dizer que um germe é $\mathscr{A}$ - determinado.

Germes de aplicações $\mathscr{K}$-determinados são chamados de tipo de singularidade finita [[16], p.201]. Usando a simetria entre a teoria de $\mathscr{A}$ - equivalência e a teoria de $\mathscr{K}$ - equivalência [veja seção 01 de [10]] sabe-se em particular que se $f \in \mathscr{O}(n, p)$ é finitamente determinado então $f$ tem tipo de singularidade finita.

Diz-se que $f$ é um germe estável se todo germe em uma vizinhança de $f$ é $\mathscr{A}$ - equivalente a $f$. Um tipo estável é uma classe de $\mathscr{A}$ - equivalência de germes estáveis. Observamos que $f$ é estável se e somente se todos os desdobramentos de $f$ são triviais. Um germe finitamente determinado $f$ tem tipo estável discreto se existe um desdobramento versal de $f$ no qual ocorre um número finito de tipos estáveis. Se o par $(n, p)$ está nas boas dimensões de Mather, então todo germe finitamente determinado $f \in \mathscr{O}(n, p)$ tem tipo estável discreto. 
Denota-se por $J(f)$ o ideal gerado pelo conjunto de menores $p \times p$ da derivada de $f$. O determinante da derivada de $f \in \mathscr{O}_{e}(n, p)$ é denotado por $J[f]$.

O teorema abaixo caracteriza geometricamente os germes finitamente determinados.

Teorema 1.6 (Gaffney). O germe de aplicação $\mathscr{K}$-finitamente determinado $f:\left(\mathbb{C}^{n}, 0\right) \rightarrow\left(\mathbb{C}^{p}, 0\right)$ é finitamente $\mathscr{A}$-determinado se e somente se para qualquer representante de $f$ existem vizinhanças $U$ de 0 em $\mathbb{C}^{n}$ e $V$ de 0 em $\mathbb{C}^{p}$, com $f(U) \subseteq V$, tais que para todos y $\neq 0$ em $V$, o multigerme $f:\left(U, f^{-1}(y) \cap \Sigma_{f}\right) \rightarrow(V, y)$ é estável.

A seguir é mostrada a caracterização geométrica dos germes estáveis, feita por Mather.

\subsection{Teorema de Mather para estabilidade}

Definição 1.7. Seja $F:\left(\mathbb{C}^{s} \times \mathbb{C}^{n},(0,0)\right) \rightarrow\left(\mathbb{C}^{s} \times \mathbb{C}^{p},(0,0)\right)$ um desdobramento versal de $f$. Dizemos que um tipo estável $\mathscr{Q}$ aparece em $F$ se para qualquer representante $F=\left(i d, f_{u}(x)\right)$ de $F$ existe um ponto $(u, y) \in \mathbb{C}^{s} \times \mathbb{C}^{p}$ tal que o germe $f_{u}:\left(\mathbb{C}^{n}, S\right) \rightarrow\left(\mathbb{C}^{p}, y\right)$ é um germe estável de tipo $\mathscr{Q}$ onde $S=f^{-1}(y) \cap \Sigma\left(f_{u}\right)$. Os pontos $(u, y)$ e $(u, x)$ com $x \in S$ são chamados de pontos de tipo estável $\mathscr{Q}$ na meta e na fonte, respectivamente.

Definição 1.8. Se $f$ é estável, denota-se o conjunto de pontos em $\mathbb{C}^{s} \times \mathbb{C}^{p}$ de tipo $\mathscr{Q}$ por $\mathscr{Q}(f)$ e $\mathscr{Q}_{S}(f)=f^{-1}(\mathscr{Q}(f))-\mathscr{Q}_{\Sigma}(f)$ onde $\mathscr{Q}_{\Sigma}(f)=f^{-1}(\mathscr{Q}(f)) \cap \Sigma(f)$.

Se fé finitamente determinado denotamos:

$$
\begin{aligned}
& \overline{\mathscr{Q}(f)}=\left(\{0\} \times \mathbb{C}^{p}\right) \cap \overline{\mathscr{Q}(F)} \\
& \overline{\mathscr{Q}_{S}(f)}=\left(\{0\} \times \mathbb{C}^{n}\right) \cap \overline{\mathscr{Q}_{S}(F)} \\
& \overline{\mathscr{Q}_{\Sigma}(f)}=\left(\{0\} \times \mathbb{C}^{n}\right) \cap \overline{\mathscr{Q}_{\Sigma}(F)}
\end{aligned}
$$

onde a barra sobre estes conjuntos significa o fecho destes.

Definição 1.9. Diz-se que $\mathscr{Q}$ é um tipo estável 0 -dimensional para o par $(n, p)$ se $\mathscr{Q}(f)$ tem dimensão 0 onde fé um representante do tipo estável $\mathscr{Q}$ em $\mathscr{O}(n, p)$.

Seja $F$ um desdobramento versal de $f$. Observamos que $\overline{\mathscr{Q}(F)}=\cap F\left(j^{p+1} F^{-1}\left(\overline{\mathscr{A} z_{i}}\right)\right)$ é um conjunto analítico fechado, onde $z_{i}$ é o $(p+1)$-jato do tipo estável $\mathscr{Q}$ e $\mathscr{A} z_{i}$ é a $\mathscr{A}$-órbita de $z_{i}$ em $J^{p+1}(n, p)$. 
Suponha que $\mathscr{Q}(F)=\left\{p_{1}, \ldots, p_{r}\right\}$ é um conjunto 0 -dimensional de pontos de tipo $\mathscr{Q}$. O invariante 0 -estável do tipo $\mathscr{Q}$ de $f$, denotado por $m(f, \mathscr{Q})$ é a multiplicidade, em $\mathscr{O}_{\overline{\mathscr{Q}}(F)},(0,0)$, do ideal $m_{s} \mathscr{O} \overline{\mathscr{Q}(F)},(0,0)$, onde $m_{s}$ denota o ideal gerado pelas coordenadas do espaço dos parâmetros $\mathbb{C}^{s}$.

Teorema 1.10 (Mather). Seja $S=\left\{x_{1}, \cdots, x_{m}\right\} \in \mathbb{C}^{n}$, e suponha que $f\left(x_{1}\right)=\cdots=f\left(x_{m}\right)=z$. Seja $A_{i}$ o germe em $x_{i}$ do conjunto $\left\{x \in \mathbb{C}^{n}\right.$ tal que o germe de fem $x$ é $\mathscr{A}$-equivalente ao germe de $f$ em $\left.x_{i}\right\}$. Então o multigerme $f:\left(\mathbb{C}^{n}, S\right) \rightarrow\left(\mathbb{C}^{p}, z\right)$ é estável se e somente se:

(i) cada germe $f:\left(\mathbb{C}^{n}, x_{i}\right) \rightarrow\left(\mathbb{C}^{p}, z\right)$ é estável, para $1 \leq i \leq m$,

(ii) o germe de aplicação $f \times \cdots \times f:\left(A_{1} \times \cdots \times A_{m},\left(x_{1}, \cdots, x_{m}\right)\right) \rightarrow\left(\mathbb{C}^{p}\right)^{m}$ é tranversal ao conjunto $\Delta_{m, p}:=\left\{\left(z_{1}, \cdots, z_{m}\right) \in\left(\mathbb{C}^{p}\right)^{m} \mid z_{i}=z_{j}\right.$ para todo $\left.i, j\right\}$.

Observamos que estes resultados são importantes para a descrição da geometria dos tipos estáveis.

\subsection{Comprimento e multiplicidades de ideais}

Definição 1.11. Uma sequência de elementos $a_{1}, \cdots, a_{d}$ de um anel A é dita regular se o ideal I gerado por $\left(a_{1}, \ldots, a_{d}\right)$ é um ideal próprio de A e a imagem de $a_{i}$ em $A /\left(a_{1}, \cdots, a_{i-1}\right)$ não é um divisor de zero para $i=1, \cdots, d$.

Definição 1.12. Seja $I=<f_{1}, \cdots, f_{d}>$ o ideal gerado por $f_{1}, \cdots, f_{d}, f_{i} \in \mathscr{O}_{n}$.

$$
V(I)=\left\{x \in \mathbb{C}^{n} ; f_{i}(x)=0, \forall i \in\{1, \cdots, d\}\right\}
$$

Dizemos que $X=V(I)$ é uma interseção completa, se o número de geradores do ideal I que define X é igual a codimensão de X. Isto significa que os geradores deste ideal formam uma sequência regular. Se X tiver uma singularidade isolada, então X é chamado de interseção completa com singularidade isolada.

O comprimento de um dado ideal $I$ de um anel analítico $R$ é definido como sendo a dim $\frac{R}{I}$, observe que isto pode ou não ser finito. A multiplicidade de um ideal $I$ é um inteiro invariante 
denotado por $e(I)$ que é definido quando $I$ tem comprimento finito.

$$
e(I)=\lim _{k \rightarrow \infty} \frac{n !}{k^{n}} \operatorname{dim}_{\mathbb{C}} \frac{R}{I^{k}}
$$

\subsection{Aplicação finita e grau}

Um germe de aplicação holomorfa $f:\left(\mathbb{C}^{n}, 0\right) \rightarrow\left(\mathbb{C}^{p}, 0\right)$ é finito se a dimensão de sua álgebra local é finita, isto é, se a multiplicidade $m(f)$ de $f$ é finita, onde

$$
m(f)=\operatorname{dim} \frac{\mathscr{O}_{n}}{f^{*}\left(m_{p}\right) \mathscr{O}_{n}}
$$

Note que para $f$ ser finito é necessário que $n \leq p$.

Um invariante que surge naturalmente no desenvolvimento da teoria é o grau, que descrevemos a seguir.

Seja $f: X \rightarrow Y$ uma aplicação analítica de espaços analíticos de mesma dimensão tal que $f(X)$ é um conjunto denso de Zariski em $Y$. Suponha que $f(x)$ é um ponto suave de $Y$ e $x$ uma componente da fibra $f^{-1}(f(x))$. Então para vizinhanças abertas $U \subset X$ de $x$ e $V \subset Y$ de $f(x)$ e um subconjunto analítico fechado $B \subset Y$ tal que:

(i) $V \backslash$ B é conexo;

(ii) $f(U) \subset V,\left.f\right|_{U}$ é própria;

(iii) $f \mid\left(U \backslash f^{-1}(B)\right)$ é $C^{\infty}$;

O número de pré-imagens em $U$ contado com a multiplicidade de qualquer ponto $y \in V \backslash B$ é chamado de grau de $f$ em $x$, denotado por $\operatorname{deg}(f)$. Observamos que o grau independe da escolha de $U, V$ e $B$.

Seja $W \subseteq\left(\mathbb{C}^{n}, 0\right)$ um germe de espaço analítico definido como o conjunto de zeros de $g_{1}, \ldots, g_{t}, g_{i} \in \mathscr{O}_{n}$ ou seja, $W=V\left(g_{1}, \ldots, g_{t}\right)$ com $\operatorname{dim}_{0} W=s$. A multiplicidade de $W$ em 0 , denotada por mult $(W)_{0}$ é definida como a multiplicidade do ideal gerado por $g_{1}, \ldots, g_{t}$. Se $\operatorname{dim}_{0} W=s$ é possível escolher uma projeção conveniente $\varphi:\left(\mathbb{C}^{n}, 0\right) \rightarrow\left(\mathbb{C}^{s}, 0\right)$ tal que

$$
\operatorname{deg}\left(\left.\varphi\right|_{W}\right)=\operatorname{mult}(W)_{0}
$$


Se $\mathscr{O}_{W}$ é Cohen-Macaulay então $\operatorname{deg}\left(\left.\varphi\right|_{W}\right)$ é o comprimento do ideal $\left\langle\varphi_{1}, \ldots, \varphi_{t}\right\rangle$ em $\mathscr{O}_{W}$, ou seja,

$$
\operatorname{deg}\left(\left.\varphi\right|_{W}\right)=\operatorname{dim} \frac{\mathscr{O}_{X, x}}{\left\langle\varphi_{1}, \ldots, \varphi_{t}, g_{1}, \ldots, g_{t}\right\rangle} .
$$

Da definição de $\operatorname{mult}(W)_{0}$ temos que se $I=<g_{1}, \ldots, g_{t}>$, então $\operatorname{mult}(W)_{0}=e(I)$. 


\section{Capítulo \\ 2 \\ Caracterização dos tipos estáveis em germes finitamente determinados}

\subsection{Os tipos estáveis em $\mathscr{O}(n, n), r$-esquemas}

Na introdução, vimos nos exemplos os tipos de singularidades 0-estáveis que aparecem no discriminante de um germe finitamente determinado. Entretanto, em vários trabalhos relacionados com a equisingularidade de famílias de aplicações tais como Gaffney [7], surgem invariantes associados aos tipos de singularidades $r$-estáveis, com $0 \leq r \leq n-1$, que estão no discriminante, no conjunto singular e também na imagem inversa do discriminante por $f$.

Neste capítulo é feita uma descrição explícita de todos os tipos estáveis na fonte e na meta para germes de coposto 1 de $\left(\mathbb{C}^{n}, 0\right)$ em $\left(\mathbb{C}^{n}, 0\right)$. Esta descrição é feita em termos dos subesquemas de pontos múltiplos de um germe $f$. Também é feito um estudo sobre a geometria destas singularidades, proposição 2.8. Na parte final deste é mostrado um algoritmo para a determinação dos tipos estáveis na fonte.

A descrição dos tipos 0-estáveis é feita em [2]. Generalizamos esta descrição para todos os tipos r-estáveis, com $0 \leq r \leq n-1$, como descrito em [1].

Dada uma aplicação contínua $f: X \rightarrow Y$ sobre um espaço analítico, o espaço de $k$-pontos múltiplos de $f$ é definido por

$$
D^{k}(f):=\text { fecho }\left\{\left(x_{1}, x_{2}, \ldots, x_{k}\right) \in X^{k}: f\left(x_{1}\right)=\ldots=f\left(x_{k}\right) \operatorname{para} x_{i} \neq x_{j}, i \neq j\right\}
$$

Todo germe de aplicação finitamente determinado $f \in \mathscr{O}(n, n)$ de coposto 1 pode ser escrito 
como

$$
f(x, z)=\left(x_{1}, \ldots, x_{n-1}, g(x, z)\right)
$$

$\operatorname{com} x=\left(x_{1}, \ldots, x_{n-1}\right) \in \mathbb{C}^{n-1}, z \in \mathbb{C}$ e $g:\left(\mathbb{C}^{n}, 0\right) \rightarrow(\mathbb{C}, 0)$ é um polinômio.

Para cada partição $\mathscr{P}=\left(r_{1}, \ldots, r_{\ell}\right)$ de um inteiro $1 \leq m \leq n$, isto é, $r_{1}+\cdots+r_{\ell}=m$, consideremos o subconjunto $D^{\ell}(f, \mathscr{P})$ de $\mathbb{C}^{n-1} \times \mathbb{C}^{\ell}$, definido por

$$
D^{\ell}(f, \mathscr{P})=\text { fecho }\left\{\left(x, z_{1}, \cdots, z_{\ell}\right) \in \mathbb{C}^{n-1} \times \mathbb{C}^{\ell}: \begin{array}{l}
z_{i} \neq z_{j}, \\
f\left(x, z_{i}\right)=f\left(x, z_{j}\right) \mathrm{e} \\
f \text { tem uma singularidade do tipo } \\
A_{r_{j}} \text { em }\left(x, z_{j}\right)
\end{array}\right\}
$$

onde 'fecho" significa o fecho analítico em $\mathbb{C}^{n-1} \times \mathbb{C}^{\ell}$. A notação $A_{r_{j}}$ é a notação de Arnold para estas singularidades.

Os conjuntos $D^{\ell}(f, \mathscr{P})$ são chamados subesquemas e são os tipos $(n-m)$-estáveis. Quando $m=n$, os subconjuntos $D^{\ell}(f, \mathscr{P})$ são chamados de zero-esquemas e são os tipos 0-estáveis definidos em [2].

A seguir é descrita a estrutura dos subesquemas ou tipos $(n-m)$-estáveis.

Próximo a um multigerme do tipo $\left(A_{r_{1}}+\cdots+A_{r_{\ell}}\right)=A_{r_{1}, \cdots, r_{\ell}}$, existem pontos na meta com $\left(r_{1}+1\right)+\left(r_{2}+1\right)+\cdots+\left(r_{\ell}+1\right)=m+\ell$ pré-imagens. Definimos um $(m+\ell)$-esquema em $\mathbb{C}^{n-1} \times \mathbb{C}^{m+\ell}$ que, sobre a diagonal apropriada, especializa-se o ideal definindo o multigerme $A_{r_{1}, \cdots, r_{\ell}}$. (Veja Lema 2.1).

As coordenadas de $\mathbb{C}^{n-1} \times \mathbb{C}^{m+\ell}$ são denotadas por

$$
(x, \mathbf{z})=\left(x, z_{0}^{1}, \cdots, z_{r_{1}}^{1}, z_{0}^{2}, \cdots, z_{r_{2}}^{2}, \cdots, z_{0}^{\ell}, \cdots, z_{r_{\ell}}^{\ell}\right)
$$

e o feixe de ideais é denotado por

$$
\mathscr{J}^{\ell}(f, \mathscr{P})=\left\langle h_{1}, h_{2}, \cdots, h_{m+\ell-1}\right\rangle \subset \mathscr{O}_{\mathbb{C}^{n-1} \times \mathbb{C}^{m+\ell}}
$$


$\operatorname{com} h_{i}$ definido por

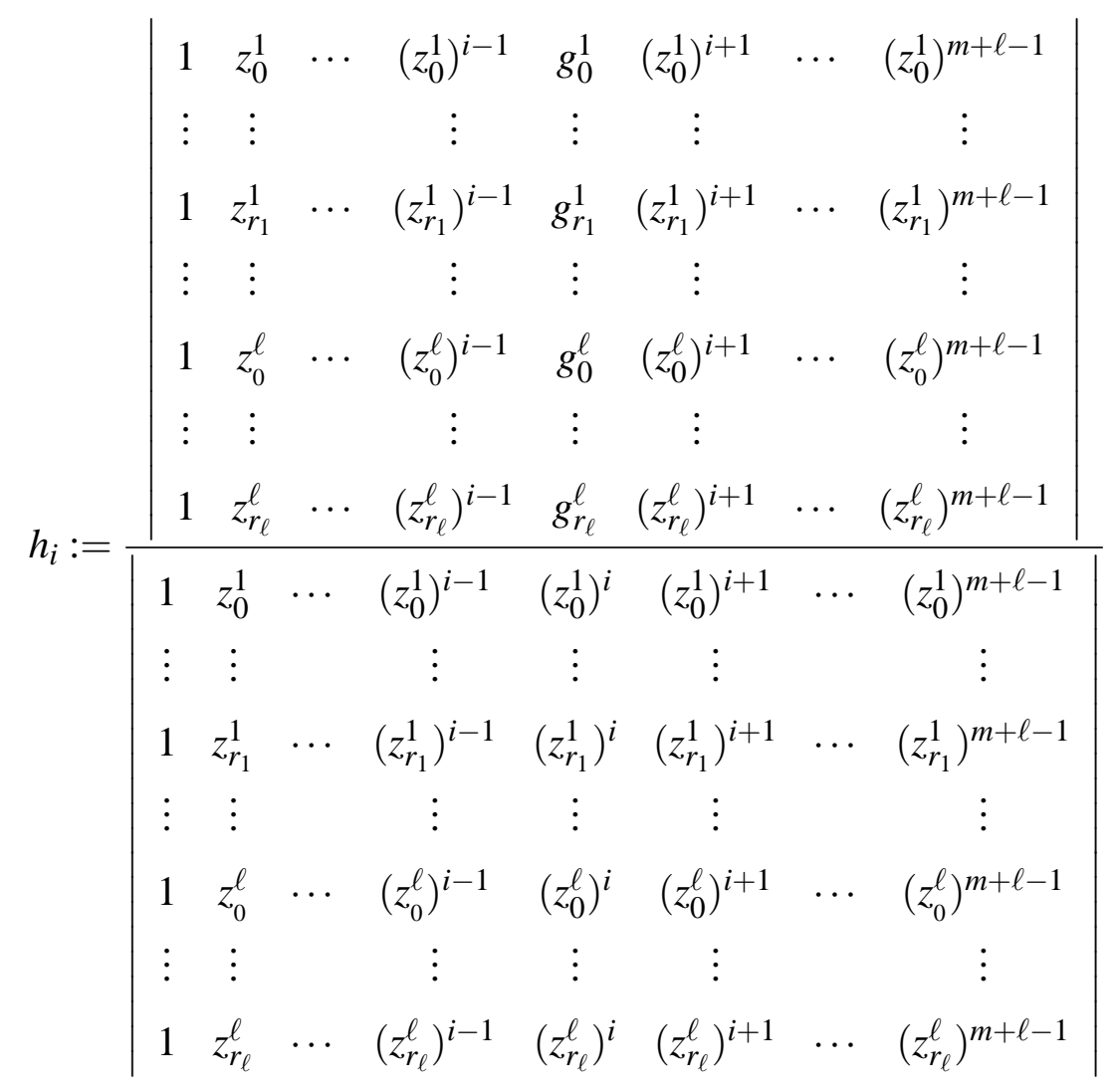

O denominador de $h_{i}$ é o determinante de Vandermonde do vetor $\left(z_{0}^{1}, \cdots, z_{r_{1}}^{1}, \cdots, z_{0}^{\ell}, \cdots, z_{r_{\ell}}^{\ell}\right)$ e $g_{i}^{k}:=g\left(x, z_{i}^{k}\right)$.

Em $\mathbb{C}^{n-1} \times \mathbb{C}^{m+\ell}$ existe uma diagonal de particular interesse, definida por,

$$
\Delta(\mathscr{P})=\left\{(x, \mathbf{z}) \in \mathbb{C}^{n-1} \times \mathbb{C}^{m+\ell} \mid z_{i}^{k}=z_{j}^{k}, \forall i, j=0, \cdots, r_{k}, \forall k=1, \cdots, \ell\right\}
$$

que pode ser parametrizada por $\left(x, z^{1}, \cdots, z^{\ell}\right)$ :

$$
(x, \mathbf{z})=\left(x, z^{1}, \cdots, z^{1}, z^{2}, \cdots, z^{2}, \cdots, z^{\ell}, \cdots, z^{\ell}\right)
$$

$\operatorname{com} z^{i}$ repetidos $r_{i}+1$ vezes.

A função cuja imagem é a diagonal $\Delta(\mathscr{P})$ é um mergulho que será denotado por

$$
j_{\ell}: \mathbb{C}^{n-1} \times \mathbb{C}^{\ell} \rightarrow \mathbb{C}^{n-1} \times \mathbb{C}^{m+\ell}
$$


Por $j_{\ell}^{*}: \mathscr{O}_{\mathbb{C}^{n-1} \times \mathbb{C}^{m+\ell}} \rightarrow \mathscr{O}_{\mathbb{C}^{n-1} \times \mathbb{C}^{\ell}}$ é denotada a sobrejeção induzida.

Seja

$$
\mathscr{I}_{\Delta(\mathscr{P})}=\left\langle z_{i}^{k}-z_{0}^{k}, i=1, \cdots, r_{k} ; k=1, \cdots, \ell\right\rangle
$$

o ideal definindo $\Delta(\mathscr{P})$. Então $j_{\ell}^{*}$ induz um isomorfismo

$$
\frac{\mathscr{O}_{\mathbb{C}^{n-1} \times \mathbb{C}^{m+\ell}}}{\mathscr{I}_{\Delta(\mathscr{P})}} \cong \mathscr{O}_{\mathbb{C}^{n-1} \times \mathbb{C}^{\ell}}
$$

Um ponto genérico de $V\left(\mathscr{I}_{\Delta(\mathscr{P})}\right)$ se escreve como na equação $2.8 \operatorname{com} z^{i} \neq z^{j}$, para $i \neq j$.

Seja $\mathscr{J}_{\Delta}^{\ell}(f, \mathscr{P})$ o feixe de ideais em $\mathscr{O}_{\mathbb{C}^{n-1} \times \mathbb{C}^{m+\ell}}$ definido por

$$
\mathscr{J}_{\Delta}^{\ell}(f, \mathscr{P}):=\mathscr{J}^{\ell}(f, \mathscr{P})+\mathscr{I}_{\Delta(\mathscr{P})}
$$

e considere o ideal $\mathscr{I}_{\Delta}^{\ell}(f, \mathscr{P}):=j_{\ell}^{*}\left(\mathscr{J}_{\Delta}^{\ell}(f, \mathscr{P})\right)$ em $\mathscr{O}_{\mathbb{C}^{n-1} \times \mathbb{C}^{\ell}}$. Então $j_{\ell}^{*}$ também induz um isomorfismo

$$
j_{\ell}^{*}: \frac{\mathscr{O}_{\mathbb{C}^{n-1} \times \mathbb{C}^{m+\ell}}}{\mathscr{J}_{\Delta}^{\ell}(f, \mathscr{P})} \rightarrow \frac{\mathscr{O}_{\mathbb{C}^{n-1} \times \mathbb{C}^{\ell}}}{\mathscr{I}_{\Delta}^{\ell}(f, \mathscr{P})}
$$

O próximo lema mostra que em um ponto genérico de $\Delta(\mathscr{P}), D^{\ell}(f, \mathscr{P})=V\left(\mathscr{J}_{\Delta}^{\ell}(f, \mathscr{P})\right)$, isto é, $f$ tem uma singularidade do tipo $A_{r_{j}}$ em $\left(x, z^{j}\right)$ e $f\left(x, z^{1}\right)=\cdots=f\left(x, z^{\ell}\right)$.

Por um abuso de notação, o subesquema $V\left(\mathscr{I}_{\Delta}^{\ell}(f, \mathscr{P})\right) \subset \mathscr{O}_{\mathbb{C}^{n-1} \times \mathbb{C}^{\ell}}$ equipado com a estrutura do feixe $\frac{\mathscr{O}_{\mathbb{C}^{n-1} \times \mathbb{C}^{\ell}}}{\mathscr{I}_{\Delta}^{\ell}(f, \mathscr{P})}$ será denotado por $D^{\ell}(f, \mathscr{P})$ também.

Lema 2.1 ([3], Lema 2.7). Em um ponto genérico de $\Delta(\mathscr{P})$ temos,

$$
\begin{aligned}
\mathscr{J}_{\Delta}^{\ell}(f, \mathscr{P})= & \left\langle\frac{\partial g}{\partial z}\left(x, z^{1}\right), \cdots, \frac{\partial^{r} 1 g}{\partial z^{r_{1}}}\left(x, z^{1}\right), \cdots, \frac{\partial g}{\partial z}\left(x, z^{\ell}\right), \cdots, \frac{\partial^{r} \ell g}{\partial z^{r} \ell}\left(x, z^{\ell}\right)\right\rangle \\
& +\left\langle g\left(x, z^{i}\right)-g\left(x, z^{1}\right) ; 2 \leq i \leq \ell\right\rangle+\mathscr{I}_{\Delta(\mathscr{P})} .
\end{aligned}
$$

Demonstração: Apêndice.

\section{Casos particulares.}

1. Observe que para a partição $\mathscr{P}=(1, \ldots, 1)$ de $m$ com $1+\ldots+1=m=\ell$, temos $D^{\ell}(f, \mathscr{P})=D^{\ell}(f)$, onde $D^{\ell}(f)$ denota o conjunto de $\ell$-múltiplos pontos de $f$. 
2. Para a partição $\mathscr{P}=\left(r_{i}\right)$, então $r_{i}=m, \ell=1$, e $D^{1}(f, \mathscr{P})=\Sigma^{1 r_{1}}(f)$, onde $\Sigma^{1 r_{1}}(f)$ é o conjunto de singularidades do tipo $\Sigma^{1, \ldots, 1}$ de $f$, de acordo com a notação de Thom-Boardmam, com $1, \ldots, 1$ repetidos $r_{i}$-vezes, i.é, $f$ tem uma singularidade do tipo $A_{r_{i}}$.

3. Considere o caso $m=n=3$ e a partição $\mathscr{P}=(2,1)$, então temos,

$$
\begin{gathered}
r_{1}=2, r_{2}=1, \ell=2 \text { e } m+\ell=5 \\
x \in \mathbb{C}^{n-1}=\mathbb{C}^{2} \Rightarrow x=\left(x_{1}, x_{2}\right) \text { e }(x, z)=\left(x_{1}, x_{2}, z_{0}^{1}, z_{1}^{1}, z_{2}^{1}, z_{0}^{2}, z_{1}^{2}\right) \in \mathbb{C}^{n-1} \times \mathbb{C}^{m+\ell}=\mathbb{C}^{2} \times \mathbb{C}^{5}
\end{gathered}
$$

Pelo lema 2.1

$$
\mathscr{J}_{\Delta}^{2}(f, \mathscr{P})=\left\langle\frac{\partial g}{\partial z}\left(x, z^{1}\right), \frac{\partial^{2} g}{\partial z^{2}}\left(x, z^{1}\right), \frac{\partial g}{\partial z}\left(x, z^{2}\right)\right\rangle+\left\langle g\left(x, z^{2}\right)-g\left(x, z^{1}\right)\right\rangle+\left\langle z_{1}^{1}-z_{0}^{1}, z_{2}^{1}-z_{0}^{1}, z_{1}^{2}-z_{0}^{2}\right\rangle
$$

Para caracterizar os tipos estáveis em $\mathscr{O}(n, n)$, precisamos dos seguintes resultados.

Definição 2.2. Sejam $D(\mathscr{P})$ o germe de aplicação de $\mathbb{C}^{n-1} \times \mathbb{C}^{m+\ell}$ em $\mathbb{C}^{m}$ dada por:

$$
D(\mathscr{P})(x, z)=\left(\frac{\partial g}{\partial z}\left(x, z^{1}\right), \ldots, \frac{\partial^{r_{1}} g}{\partial z^{r_{1}}}\left(x, z^{1}\right), \ldots, \frac{\partial g}{\partial z}\left(x, z^{\ell}\right), \ldots, \frac{\partial^{r_{\ell}} g}{\partial z^{r_{\ell}}}\left(x, z^{\ell}\right)\right)
$$

e $R(\mathscr{P})$ o germe de aplicação de $\mathbb{C}^{n-1} \times \mathbb{C}^{m+\ell}$ em $\mathbb{C}^{\ell-1}$ por:

$$
R(\mathscr{P})(x, z)=\left(g\left(x, z^{2}\right)-g\left(x, z^{1}\right), g\left(x, z^{3}\right)-g\left(x, z^{1}\right), \ldots, g\left(x, z^{\ell}\right)-g\left(x, z^{1}\right)\right)
$$

Definição 2.3. Sejam $H^{\ell}$ o germe de aplicação de $\mathbb{C}^{n-1} \times \mathbb{C}^{m+\ell}$ em $\mathbb{C}^{m+\ell-1}$ definido pelos geradores $h_{i}$ do ideal $\mathscr{J}^{\ell}(f, \mathscr{P})$, ou seja,

$$
H^{\ell}(x, z)=\left(h_{1}(x, z), \ldots, h_{\ell+m-1}(x, z)\right)
$$

e $E(\mathscr{P})$ o germe de aplicação de $\mathbb{C}^{n-1} \times \mathbb{C}^{m+\ell}$ em $\mathbb{C}^{m}$ definido pelos geradores do ideal $\mathscr{I}_{\Delta(\mathscr{P})}$, isto é,

$$
E(\mathscr{P})(x, z)=\left(z_{1}^{1}-z_{0}^{1}, \cdots, z_{r_{1}}^{1}-z_{0}^{1}, \ldots, z_{1}^{\ell}-z_{0}^{\ell}, \ldots, z_{r_{\ell}}^{\ell}-z_{0}^{\ell}\right)
$$


$e M: \mathbb{C}^{n-1} \times \mathbb{C}^{m+\ell} \rightarrow \mathbb{C}^{m+\ell-1} \times \mathbb{C}^{m}$, dado por

$$
M(x, z)=\left(H^{\ell}(x, z), E(\mathscr{P})(x, z)\right)
$$

Lema 2.4 ([3], Lema 2.10.). Seja G um grupo finito agindo linearmente sobre um espaço vetorial $V$, e seja $F: V \rightarrow W$ uma aplicação $G$-invariante. Seja H um subgrupo de G, e suponha que o ponto $x_{0} \in V$ permanece no FixH, que é o conjunto de $x \in V$ fixado por todos $h \in H$. Então $F$ é uma submersão em $x_{0}$ se e somente se F| FixH é uma submersão em $x_{0}$.

Proposição 2.5 ([3], Proposição 2.13). Seja $f(x, z)=(x, g(x, z)), x=\left(x_{1}, \ldots, x_{n-1}\right) \in \mathbb{C}^{n-1}, z \in$ $\mathbb{C}$, seja $m=r_{1}+\cdots+r_{\ell}, \mathscr{P}=\left(r_{1}, \cdots, r_{\ell}\right)$ partição de $m$. Suponha que $f\left(x, z_{0}^{1}\right)=\cdots=$ $f\left(x, z_{r_{1}}^{1}\right)=\cdots=f\left(x, z_{0}^{\ell}\right)=\cdots=f\left(x, z_{r_{\ell}}^{\ell}\right)=w$, com $z_{i}^{k} \neq z_{j}^{k}, i, j=0, \ldots, r_{k}, k=1, \ldots, \ell$, com $i \neq j$. Suponha que $f$ é do tipo $\Sigma^{1_{r_{k}-1}, 0}$ em $\left(x, z_{i}^{k}\right)$. Então, se $z=\left(z^{1}, \ldots, z^{1}, \ldots, z^{\ell}, \ldots, z^{\ell}\right)$, com $z^{k}$ repetidos $r_{k}$-vezes, $k=1, \ldots, \ell$ e $S=\left\{\left(x, z^{1}\right), \ldots,\left(x, z^{\ell}\right)\right\}$, são equivalentes:

(i) $f:\left(\mathbb{C}^{n}, S\right) \rightarrow\left(\mathbb{C}^{n}, w\right)$ é estável,

(ii) A aplicação $H^{\ell}$ definindo $D^{k}(f)$ é uma submersão em $(x, z)$,

(iii) A aplicação M é uma submersão em $(x, z)$.

Observação 2.6. O grupo simétrico $S_{m+\ell}$ age sobre $\mathbb{C}^{n-1+m+\ell}$ por permutação em suas últimas $m+\ell$ coordenadas e sobre $\mathbb{C}^{m+\ell}$ por permutação em todas as suas coordenadas. O grupo simétrico $G=S_{r_{1}} \times \ldots \times S_{r_{\ell}}$ age sobre $\mathbb{C}^{m+\ell}$, onde cada $S_{r_{i}}$ age sobre as coordenadas $z_{0}^{i}, \ldots, z_{r_{i}}^{i}$.

Demonstração: $(i i i) \Leftrightarrow(i i)$ Esta equivalência é uma aplicação imediata do lema 2.4. De fato, se $G=S_{r_{1}} \times \ldots \times S_{r_{\ell}}$, então $H^{\ell}$ é $G$-invariante pois $(x, z) \in F i x G$. Pelo Lema 2.4 , $H^{\ell}$ é uma submersão em $(x, z)$ se e somente se $\left.H^{\ell}\right|_{F i x G}$ é uma submersão em $(x, z)$. Como $F i x G=\Delta(\mathscr{P})$, e $\Delta(\mathscr{P})$ é definido pela submersão $E(\mathscr{P})$, a conclusão segue.

$(i) \Leftrightarrow($ iii) Pelo lema 2.1, o item (iii) é equivalente a restrição

$$
(D(\mathscr{P}), R(\mathscr{P})): \Delta(\mathscr{P}) \rightarrow \mathbb{C}^{m+\ell-1}
$$

ser uma submersão em $(x, z)$. Agora, 


$$
D(\mathscr{P})^{-1}(0) \cap \Delta(\mathscr{P}) \cong \prod_{i=1}^{\ell} \Sigma^{1_{r_{i}-1}}(f) \cap \Delta_{x}
$$

onde

$$
\Delta_{x}=\left\{\left(x^{1}, z^{1}\right), \ldots,\left(x^{\ell}, z^{\ell}\right) \in\left(\mathbb{C}^{n}\right)^{\ell} / x^{i}=x^{j} \quad \forall i, j, x^{i} \in \mathbb{C}^{n-1}\right\}
$$

e também

$$
\left(D(\mathscr{P})^{-1}(0) \cap \Delta(\mathscr{P}) \cap R(\mathscr{P})^{-1}(0) \cong \prod_{i=1}^{\ell} \Sigma^{1_{r_{i}-1}}(f) \cap(f \times \ldots \times f)^{-1}\left(\Delta_{\ell, n}\right) \cap \Delta_{x}\right.
$$

com

$$
\Delta_{\ell, n}=\left\{\left(w_{1}, \cdots, w_{\ell}\right) \in\left(\mathbb{C}^{n}\right)^{\ell} \mid w_{i}=w_{j}, w_{i} \in \mathbb{C}^{n}, \text { para todo } i, j\right\}
$$

Segue que

$$
(D(\mathscr{P}), R(\mathscr{P})): \Delta(\mathscr{P}) \rightarrow \mathbb{C}^{m+\ell-1}
$$

é uma submersão em $(x, z)$ se e somente se $\Sigma^{1_{r_{i}-1}}(f)$ é uma submersão em $\left(x, z_{i}^{k}\right)$, para $i=$ $0, \ldots, r_{k}$, e $j=0, \ldots, \ell$ e a restrição

$$
f \times \ldots \times f: \Sigma^{1_{r_{1}-1}}(f) \times \ldots \times \Sigma^{1_{r_{\ell}-1}}(f) \rightarrow\left(\mathbb{C}^{n}\right)^{\ell}
$$

for transversal a $\Delta_{\ell, n}$. Pelo Teorema 1.10 , isto é equivalente a $(i)$, pois $A_{i}=\Sigma^{1 r_{i}-1}(f)$.

O exemplo abaixo ilustra a construção do isomorfismo 2.13 da demonstração do teorema acima.

Exemplo 2.7. Considerando o exemplo 3. dos casos particulares do lema 2.1, sejam $m=n=3$ e $\mathscr{P}=(2,1)$, pela igualdade 2.7 ,

$$
\Delta(\mathscr{P})=\left\{\left(x, z^{1}, z^{1}, z^{1}, z^{2}, z^{2}\right)\right\} \subset \mathbb{C}^{2} \times \mathbb{C}^{5}
$$

pela definição $2.2, D(\mathscr{P}): \mathbb{C}^{2} \times \mathbb{C}^{5} \rightarrow \mathbb{C}^{3}$ é dado por

$$
D(\mathscr{P})(x, z)=\left(\frac{\partial g}{\partial z}\left(x, z^{1}\right), \frac{\partial^{2} g}{\partial z^{2}}\left(x, z^{1}\right), \frac{\partial g}{\partial z}\left(x, z^{2}\right)\right)
$$

e $D(\mathscr{P})(x, z)=(0,0,0)$ se e somente se, 


$$
\frac{\partial g}{\partial z}\left(x^{1}, z^{1}\right)=\frac{\partial^{2} g}{\partial z^{2}}\left(x^{1}, z^{1}\right)=0 \quad \text { e } \quad \frac{\partial g}{\partial z}\left(x^{2}, z^{2}\right)=0
$$

$\operatorname{com} x^{1}=\left(x_{1}^{1}, x_{2}^{1}\right)$ e $x^{2}=\left(x_{1}^{2}, x_{2}^{2}\right)$, então

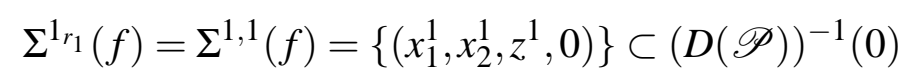

e

$$
\Sigma^{1_{r_{2}}}(f)=\Sigma^{1}(f)=\left\{\left(x_{1}^{2}, x_{2}^{2}, 0, z^{2}\right)\right\} \subset(D(\mathscr{P}))^{-1}(0)
$$

$(D(\mathscr{P}))^{-1}(0) \subset \mathbb{C}^{n-1} \times \mathbb{C}^{\ell}=\mathbb{C}^{2} \times \mathbb{C}^{2}$

Consideramos aqui $x^{1} \neq x^{2}$ no lugar de $x$ para obtermos os conjuntos $\Sigma^{1_{r_{1}}}(f)$ e $\Sigma^{1_{r_{2}}}(f)$

No caso geral,

$$
\Sigma^{1_{r_{i}}}(f)=\Sigma^{1, \ldots, 1}(f)=\left\{\left(x_{1}^{i}, x_{2}^{i}, \ldots, x_{n-1}^{i}, 0, \ldots, 0, z^{i}, 0, \ldots, 0\right)\right\} \subset(D(\mathscr{P}))^{-1}(0) \subset \mathbb{C}^{n-1} \times \mathbb{C}^{\ell}
$$

$\operatorname{com} z^{i}$ na $(n-1+i)$-ésima posição.

Neste exemplo,

$$
\Delta_{x}=\left\{\left(x^{1}, z^{1}\right),\left(x^{2}, z^{2}\right) \in\left(\mathbb{C}^{3}\right)^{2} / x^{1}=x^{2}, x^{i} \in \mathbb{C}^{2}, i=1,2\right\}
$$

Temos que

$$
\Sigma^{1^{r_{1}}}(f) \times \Sigma^{1_{r_{2}}}(f)=\left\{\left(x_{1}^{1}, x_{2}^{1}, z^{1}, 0\right)\right\} \times\left\{\left(x_{1}^{2}, x_{2}^{2}, 0, z^{2}\right)\right\} \subset\left(\mathbb{C}^{2} \times \mathbb{C}^{2}\right) \times\left(\mathbb{C}^{2} \times \mathbb{C}^{2}\right)
$$

mais ainda,

$$
\left\{\left(x_{1}^{1}, x_{2}^{1}, z^{1}, 0\right)\right\} \times\left\{\left(x_{1}^{2}, x_{2}^{2}, 0, z^{2}\right)\right\} \subset\left(\mathbb{C}^{2} \times \mathbb{C} \times\{0\}\right) \times\left(\mathbb{C}^{2} \times\{0\} \times \mathbb{C}\right) \cong\left(\mathbb{C}^{2} \times \mathbb{C}\right) \times\left(\mathbb{C}^{2} \times \mathbb{C}\right)
$$

então,

$$
\Sigma^{1_{r_{1}}}(f) \times \Sigma^{1_{r_{2}}}(f) \cong\left\{\left(x_{1}^{1}, x_{2}^{1}, x_{1}^{2}, x_{2}^{2}, z^{1}, z^{2}\right)\right\} \subset\left(\mathbb{C}^{2} \times \mathbb{C}^{2}\right) \times \mathbb{C}^{2}
$$

Logo a intersecção do isomorfismo $2.14 \operatorname{com} \Delta_{x}$ fica,

$$
\Sigma^{1^{r_{1}}}(f) \times \Sigma^{1_{r_{2}}}(f) \cap \Delta_{x} \cong\left\{\left(x_{1}, x_{2}, z^{1}, z^{2}\right)\right\} \subset \mathbb{C}^{4}
$$


onde, $x_{1}:=x_{1}^{1}=x_{1}^{2}, x_{2}:=x_{2}^{1}=x_{2}^{2}$

portanto,

$$
D(\mathscr{P})^{-1}(0) \cap \Delta(\mathscr{P}) \cong \Sigma^{1 r_{1}}(f) \times \Sigma^{1_{r_{2}}}(f) \cap \Delta_{x}
$$

O próximo resultado determina a relação entre a estabilidade do germe $f$ e a geometria das variedades $V\left(\mathscr{J}_{\Delta}^{\ell}(f, \mathscr{P})\right)$.

Proposição 2.8 ([1], Proposição 3.2). Seja $f:\left(\mathbb{C}^{n}, 0\right) \rightarrow\left(\mathbb{C}^{n}, 0\right)$ um germe de aplicação finitamente determinado de coposto 1. Então, para qualquer partição $\mathscr{P}=\left(r_{1}, \ldots, r_{\ell}\right)$ de $m \leq n$ temos:

(i) f é estável se e somente se $V\left(\mathscr{J}_{\Delta}^{\ell}(f, \mathscr{P})\right)$ é suave de dimensão $n-m$, ou vazia;

(ii) O ideal $\mathscr{J}_{\Delta}^{\ell}(f, \mathscr{P})$ em 0 ou é uma ICIS de dimensão $n-m$, ou é vazia;

Demonstração: (i) Temos pela proposição 2.5, que $f$ é estável do tipo $A_{r_{i}}$ em 0 , se e somente se o germe de aplicação $M$ é uma submersão. Neste caso $M^{-1}(0)=V\left(\mathscr{J}_{\Delta}^{\ell}(f, \mathscr{P})\right.$ e como 0 é subvaridade de codimensão $2 m+\ell-1, V\left(\mathscr{J}_{\Delta}^{\ell}(f, \mathscr{P})\right)$ também é uma subvariedade suave de codimensão $2 m+\ell-1$, e portanto de dimensão $n-m$.

(ii) Suponha que $f$ é finitamente determinado e escolha representantes $f: U \rightarrow V$ como no Teorema 1.10, critério geométrico de Mather-Gaffney. Mostraremos que para qualquer partição $\mathscr{P}$ de um $m \leq n$ e em qualquer ponto $(x, \mathbf{z}) \neq(0,0)$ a aplicação $M$ é uma submersão. Se necessário restringimos $U$ tal que $f$ é uma singularidade do tipo $A_{r_{1}, \ldots, r_{\ell}}$, depois de reordenar se necessário, podemos supor que $(x, \mathbf{z})$ é um ponto genérico de $\mathscr{I}_{\Delta(\mathscr{P})}$ para alguma partição $\mathscr{P}=\left(r_{1}, \ldots, r_{\ell}\right)$, portanto $(x, \mathbf{z})=\left(x, z^{1}, \cdots, z^{1}, z^{2}, \cdots, z^{2}, \cdots, z^{\ell}, \cdots, z^{\ell}\right)$. Agora supomos que $f$ é uma singularidade do tipo $A_{r_{i}}$ em $\left(x, z^{i}\right)$. Segue pelo critério Geométrico que o multigerme de $f$ em $\left\{\left(x, z^{1}\right), \ldots,\left(x, z^{\ell}\right)\right\}$ é estável, então pela proposição 2.5 a aplicação $M$ é uma submersão. Assim para qualquer ponto distinto da origem, as $2 m+\ell-1$ funções gerando $\mathscr{J}_{\Delta}^{\ell}(f, \mathscr{P})$ definem uma submersão. Como a codimensão de $V\left(\mathscr{J}_{\Delta}^{\ell}(f, \mathscr{P})\right)$ é igual a $2 m+\ell-1$ que coincide com o número de geradores de $\mathscr{J}_{\Delta}^{\ell}(f, \mathscr{P})$, segue que $V\left(\mathscr{J}_{\Delta}^{\ell}(f, \mathscr{P})\right)$ é uma ICIS em 0 de dimensão $n-m$.

Como consequência desta proposição e o isomorfismo dado em 2.12, obtemos o seguinte corolário: 
Corolario 2.9. Seja $f:\left(\mathbb{C}^{n}, 0\right) \rightarrow\left(\mathbb{C}^{n}, 0\right)$ um germe de aplicação de coposto 1 . Então, para qualquer partição $\mathscr{P}=\left(r_{1}, \ldots, r_{\ell}\right)$ de $m \leq n$, o germe de $D^{\ell}(f, \mathscr{P}) \subset \mathbb{C}^{n-1} \times \mathbb{C}^{\ell}$ em 0 é uma ICIS de dimensão $n-m$.

Será mostrado agora a descrição explícita dos tipos estáveis na fonte e na meta de qualquer germe de aplicação finitamente determinado $f \in \mathscr{O}(n, n)$ de coposto 1 . Para isto usamos a seguinte notação.

Para cada partição $\mathscr{P}=\left(r_{1}, \ldots, r_{\ell}\right)$ de $m \leq n$, denotamos por $D_{1}^{\ell}(f, \mathscr{P})$ a projeção de $D^{\ell}(f, \mathscr{P})$ para o $(x, z)$-espaço. Lembramos que cada um destes conjuntos $D_{1}^{\ell}(f, \mathscr{P})$ é um subconjunto de $\Sigma(f)$.

Exemplo 2.10. Para germes de aplicações finitamente determinados $f \in \mathscr{O}(2,2)$ de coposto 1 , as possíveis partições de 1 e 2 são: para $m=1$ só temos a partição (1) e para $m=2$ temos duas partições $(1,1)$ e (2). Então,

1. $D_{1}^{1}(f,(1))=\Sigma(f)$ é o conjunto singular de $f$,

2. $D_{1}^{2}(f,(1,1))=D^{2}(f)$ é o conjunto de pontos duplos de $f$,

3. $D_{1}^{1}(f,(2))=\Sigma^{1,1}(f)$ é o conjunto de cúspides de $f$.

Para germes de aplicações finitamente determinados $f \in \mathscr{O}(3,3)$ de coposto 1 , as possíveis partições de 1, 2 e 3 são: para $m=1$ temos a partição $(1)$, para $m=2$ temos $(1,1)$ e $(2)$ e para $m=3$ as partições são $(2,1),(1,1,1)$ e $(3)$. Então,

1. $D_{1}^{1}(f,(1))=\Sigma(f)$ é o conjunto singular de $f$,

2. $D_{1}^{2}(f,(1,1))=D^{2}(f)$ é a curva de pontos duplos de $f$,

3. $D_{1}^{1}(f,(2))=\Sigma^{1,1}(f)$ é a curva cuspidal,

4. $D^{3}(f,(1,1,1))=D^{3}(f)$ é o conjunto de pontos triplos,

5. $D_{1}^{1}(f,(3))=\Sigma^{1,1,1}(f)$ é o conjunto de rabos de andorinha de $f$.

No próximo teorema serão usadas as seguintes notações.

Seja

$$
X(f):=\overline{\left(f^{-1}(\Delta(f))-\Sigma(f)\right)}
$$


Para cada partição $\mathscr{P}$ de $m \leq n$, é definido o conjunto $X_{1}^{\ell}(f, \mathscr{P})$ por,

$$
X_{1}^{\ell}(f, \mathscr{P}):=f^{-1}\left(f\left(D_{1}^{\ell}(f, \mathscr{P})\right)\right)-\left(D_{1}^{\ell}(f, \mathscr{P}) \cap \Sigma(f)\right)
$$

Finalmente no teorema abaixo são descritos completamente os tipos estáveis.

Teorema 2.11 ([1], Teorema 3.5). Seja $f \in \mathscr{O}(n, n)$ germe de aplicação finitamente determinado de coposto 1. Então,

(i) Os tipos estáveis na fonte $s \tilde{a} o D_{1}^{\ell}(f, \mathscr{P}) \subset \Sigma(f)$ e $X_{1}^{\ell}(f, \mathscr{P}) \subset X(f)$, para todas partições $\mathscr{P}=\left(r_{1}, \ldots, r_{\ell}\right)$ de todo $m \leq n$.

(ii) Os tipos estáveis na meta são $f\left(D_{1}^{\ell}(f, \mathscr{P})\right) \subset \Delta(f)$, para todas partições $\mathscr{P}=\left(r_{1}, \ldots, r_{\ell}\right)$ de cada $m \leq n$.

(iii) As dimensões de $X_{1}^{\ell}(f, \mathscr{P})$ e de $f\left(D_{1}^{\ell}(f, \mathscr{P})\right)$ são ambas $n-m$.

Demonstração: $(i)$ Um germe de aplicação estável $f \in \mathscr{O}(n, n)$ tem uma singularidade do tipo $A_{k}$ se é $\mathscr{A}$-equivalente ao germe

$$
\left(x_{1}, \ldots, x_{n-1}, z\right) \rightarrow\left(x_{1}, \ldots, x_{n-1}, z^{k+1}+x_{1} z^{k-1}+\ldots+x_{k-1} z\right)
$$

Além disso, qualquer germe de aplicação estável de coposto 1 é uma $A_{k}$ singularidade para algum número natural $k$, portanto o conjunto de pontos em $\mathbb{C}^{n}$ onde uma aplicação estável tem uma $A_{k}$ singularidade é uma subvariedade suave de codimensão $k$. A imagem deste conjunto por $f$ também é uma subvariedade suave de codimensão $k$. Como $f$ é finitamente determinado, segue pelo critério Geométrico de Mather-Gaffney, que existem vizinhanças $U$ e $V$ de 0 em $\mathbb{C}^{n}$ tais que $f^{-1}(0) \cap U \cap \Sigma(f)=0$ e para cada $y \in V, y \neq 0$, o germe $f:\left(\mathbb{C}^{n}, S\right) \rightarrow\left(\mathbb{C}^{n}, y\right)$ é estável ( $S=f^{-1}(y) \cap U \cap \Sigma(f)$ ), portanto para cada $x \in S$, o germe $f:\left(\mathbb{C}^{n}, x\right) \rightarrow\left(\mathbb{C}^{n}, y\right)$ é uma singularidade $A_{k}$ para algum $k$ e estas subvariedades no discriminante estão em posição geral. Mas isto ocorre se e somente se $r_{1}+r_{2}+\ldots . .+r_{j}=m \leq n$. O resultado segue do lema 2.1. Estes multigermes são chamados de singularidades de tipo $A_{\mathscr{P}}$.

(ii) e (iii) Do corolário dado na página 19 de [6], sabemos que existem vizinhanças da origem $U_{1}$ em $\mathbb{C}^{n-1} \times \mathbb{C}^{\ell}$ e $U_{2}$ em $\mathbb{C}^{n}$ tais que a aplicação $p: D^{\ell}(f, \mathscr{P}) \rightarrow U_{2}$ induzida pela projeção 
$U_{1} \rightarrow U_{2}$ é própria e finita. Como $f$ é própria e finita, a aplicação $f \circ p$ também é própria e finita, então $V=(f \circ p)\left(D^{\ell}(f, \mathscr{P})\right)$ é uma subvariedade analítica $n-m$-dimensional, em particular, $f\left(D_{1}^{\ell}(f, \mathscr{P})\right)$ é $n-m$-dimensional. Como $D_{1}^{\ell}(f, \mathscr{P})$ é $n-m$-dimensional e $f$ é própria e finita, também obtemos que $X_{1}^{\ell}(f, \mathscr{P})$ e um espaço analítico de dimensão $n-m$.

As seguintes proposições descrevem a estrutura dos conjuntos $D_{1}^{\ell}(f, \mathscr{P}) \subset \Sigma(f)$.

Proposição 2.12 ([1], Proposição 3.6). Seja $p_{\ell}: \mathbb{C}^{n-1} \times \mathbb{C}^{\ell} \rightarrow \mathbb{C}^{n}$ a projeção

$$
\left(x_{1}, \ldots, x_{n-1}, z_{1}, \ldots, z_{\ell}\right) \mapsto\left(x_{1}, \ldots, x_{n-1}, z_{1}\right)
$$

e $p_{\ell}^{*}$ a sobrejeção associada. Seja $\mathscr{I}_{1}^{\ell}(f, \mathscr{P}):=\left(p_{\ell}^{*}\right)^{-1}\left(\mathscr{I}_{\Delta}^{\ell}(f, \mathscr{P})\right)$. Então $p_{\ell}^{*}$ induz um isomorfismo

$$
p_{\ell}^{*}: \frac{\mathscr{O}_{n}}{\mathscr{I}_{1}^{\ell}(f, \mathscr{P})} \rightarrow \frac{\mathscr{O}_{n-1+\ell}}{\mathscr{I}_{\Delta}^{\ell}(f, \mathscr{P})}
$$

Para cada partição $\mathscr{P}=\left(r_{1}, \ldots r_{\ell}\right)$ de $m \leq n$, o conjunto $D_{1}^{\ell}(f, \mathscr{P})$ é uma ICIS.

Demonstração: Como $p_{\ell}^{*}$ é sobrejetivo, mostraremos que é injetivo. Suponhamos que não é injetivo, então existe um elemento não nulo $g \in \frac{\mathscr{O}_{n}}{\mathscr{I}_{1}^{\ell}(f, \mathscr{P})}$ tal que $p_{\ell}^{*}(g)=0$, portanto $g_{\mid p_{\ell}\left(D^{\ell}(f, \mathscr{P})\right)}=$ 0 e $p_{\ell}\left(D^{\ell}(f, \mathscr{P})\right) \subset g^{-1}(0) \subset V\left(\mathscr{I}_{\Delta}^{\ell}(f, \mathscr{P})\right)$, mas $p_{\ell}\left(D^{\ell}(f, \mathscr{P})\right)=D_{1}^{\ell}(f, \mathscr{P})$ por definição, logo $D_{1}^{\ell}(f, \mathscr{P}) \subset V\left(\mathscr{I}_{\Delta}^{\ell}(f, \mathscr{P})\right)$. Observe que $\mathscr{I}_{1}^{\ell}$ é o ideal que define $D_{1}^{\ell}(f, \mathscr{P})$ então $V\left(\mathscr{I}_{1}^{\ell}(f, \mathscr{P})\right)=$ $D_{1}^{\ell}(f, \mathscr{P}) \log , V\left(\mathscr{I}_{1}^{\ell}(f, \mathscr{P})\right) \subset V\left(\mathscr{I}_{\Delta}^{\ell}(f, \mathscr{P})\right)$ e isto implica que $\left.\mathscr{I}_{\Delta}^{\ell}(f, \mathscr{P})\right) \subset \mathscr{I}_{\Delta}^{1}(f, \mathscr{P})$. Contradição, pois $V\left(\mathscr{I}_{1}^{\ell}(f, \mathscr{P})\right) \subset V\left(\mathscr{I}_{\Delta}^{\ell}(f, \mathscr{P})\right)$. Portanto, $p_{\ell}^{*}$ é isomorfismo.

Então, do lema 1.8 de [8], concluímos que se

$$
d\left(\mathscr{I}_{\Delta}^{\ell}(f, \mathscr{P})\right) \text { e } d\left(\left(p_{\ell}^{*}\right)^{-1}\left(\mathscr{I}_{\Delta}^{\ell}(f, \mathscr{P})\right)\right)
$$

denotam o número mínimo de geradores de $\mathscr{I}_{\Delta}^{\ell}(f, \mathscr{P})$ e $\left(p_{\ell}^{*}\right)^{-1}\left(\mathscr{I}_{\Delta}^{\ell}(f, \mathscr{P})\right)$ respectivamente, portanto

$$
n-1-\ell-d\left(\mathscr{I}_{\Delta}^{\ell}(f, \mathscr{P})\right)=n-d\left(\left(p_{\ell}^{*}\right)^{-1}\left(\mathscr{I}_{\Delta}^{\ell}(f, \mathscr{P})\right)\right),
$$

$\mathrm{e}$

$$
d\left(\left(p_{\ell}^{*}\right)^{-1}\left(\mathscr{I}_{\Delta}^{\ell}(f, \mathscr{P})\right)\right)=m
$$


Escolhemos uma projeção $p_{\ell}$ que é finita. Então,

$$
p_{\ell}\left(V\left(\mathscr{I}_{\Delta}^{\ell}(f, \mathscr{P})\right)\right)=V\left(\left(p_{\ell}^{*}\right)^{-1}\left(\mathscr{I}_{\Delta}^{\ell}(f, \mathscr{P})\right)\right)=D_{1}^{\ell}(f, \mathscr{P})
$$

é de dimensão $n-m$. Como o ideal $\left(p_{\ell}^{*}\right)^{-1}\left(\mathscr{I}_{\Delta}^{\ell}(f, \mathscr{P})\right)=\mathscr{I}_{1}^{\ell}(f, \mathscr{P})$ que define $D_{1}^{\ell}(f, \mathscr{P})$ tem $m$ geradores, obtemos que $D_{1}^{\ell}(f, \mathscr{P})$ é uma I.C.I.S.

\subsection{Algoritmo para os tipos estáveis na fonte}

Nesta seção é descrito um algoritmo que computa os ideais que definem os tipos estáveis na fonte para todas partições $\mathscr{P}=\left(r_{1}, \ldots, r_{\ell}\right)$ de $m \leq n$, no qual é uma generalização do caso $m=n$ do algoritmo dado em [18]. Foi implementado usando o software Maple. Uma versão anterior deste algoritmo é encontrado em [1].

Abaixo segue o algoritmo.

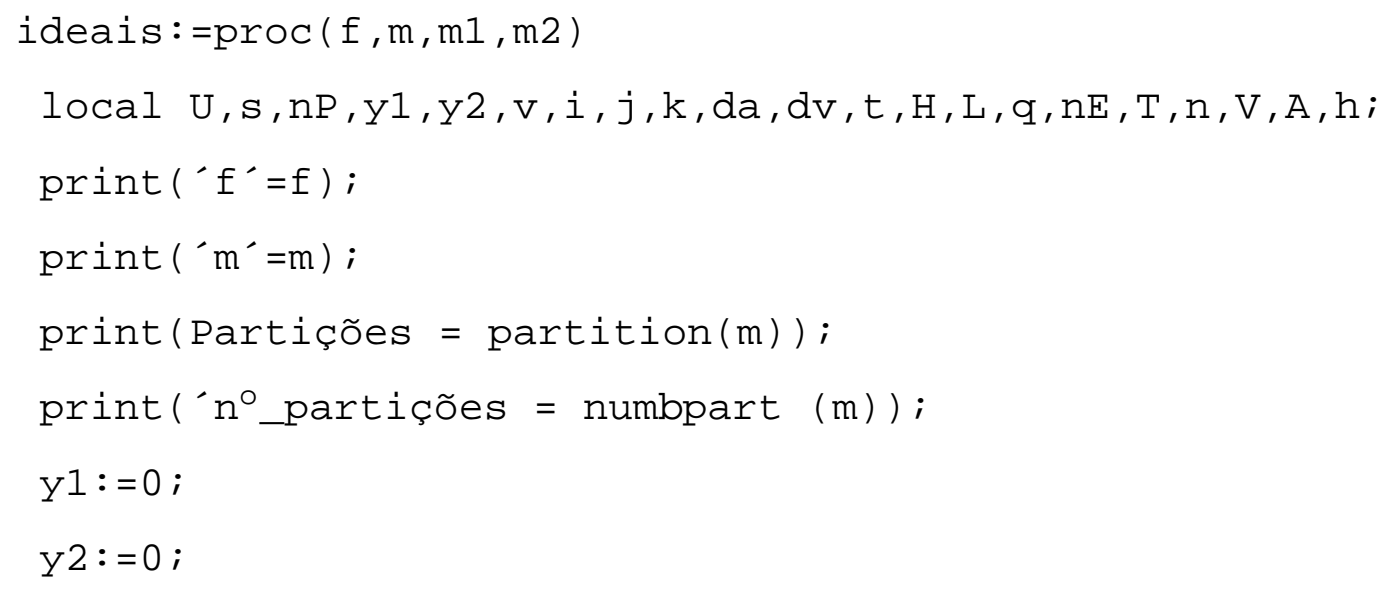

Na primeira parte do algoritmo, que corresponde da linha 1 até a linha 8, estão definidas as variáveis locais

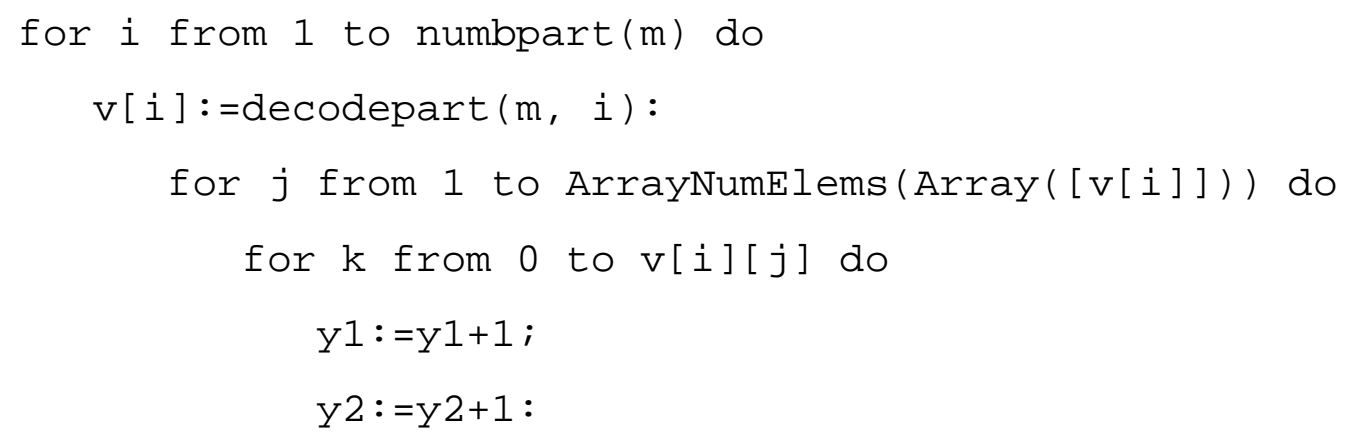




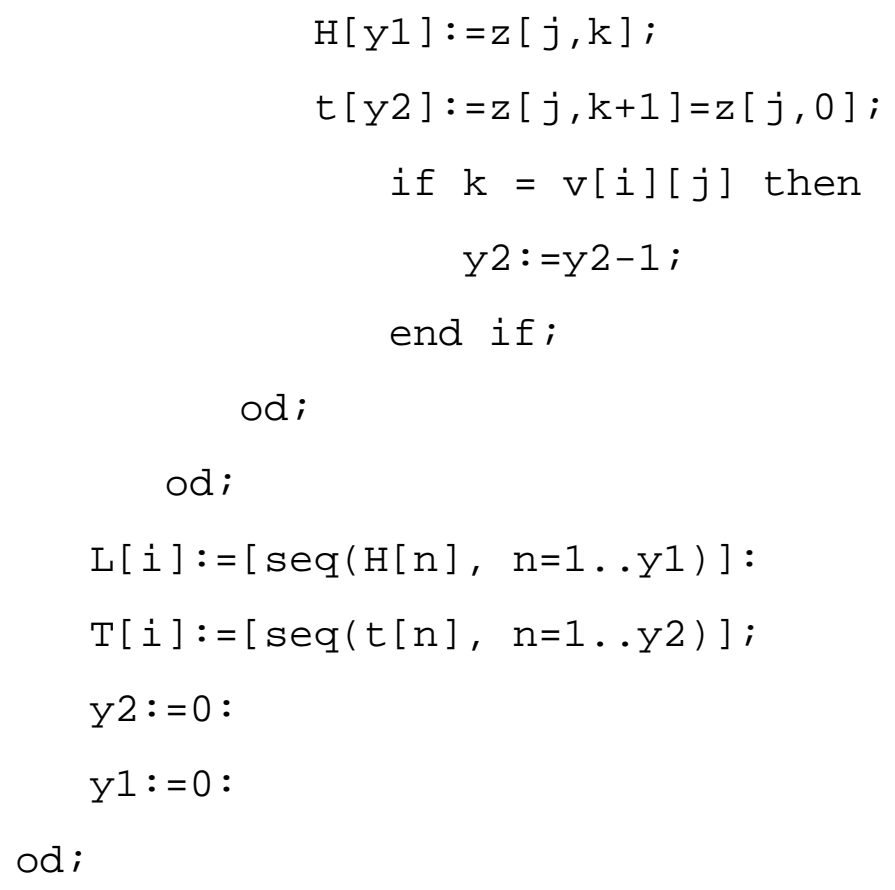

A rotina entre as linhas 9 e 27 é responsável pela construção e organização das variáveis z[j,k] de acordo com cada partição, para que possam ser utilizadas corretamente.

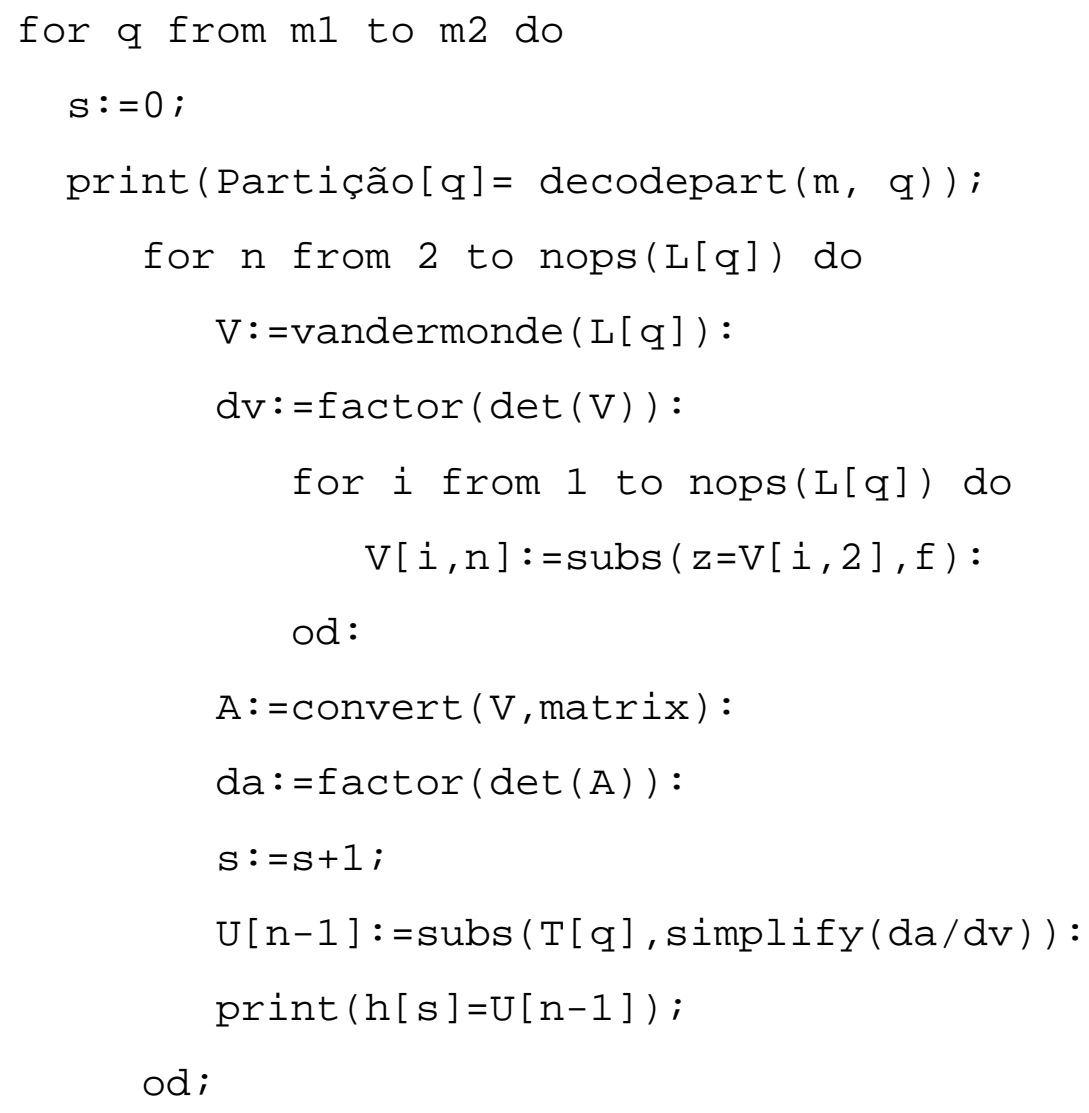


od;

end:

A última parte do algoritmo, a parte principal, faz os cálculos para a determinação dos ideais que definem os tipos estáveis na fonte. Mais especificamente, calcula o feixe de ideais

$$
\mathscr{J}^{\ell}(f, \mathscr{P})=\left\langle h_{1}, h_{2}, \cdots, h_{m+\ell-1}\right\rangle \subset \mathscr{O}_{\mathbb{C}^{n-1} \times \mathbb{C}^{m+\ell}}
$$

onde cada $h_{i}$ é obtido pelo quociente dado em 2.6

\section{Descrição das principais variáveis locais utilizadas.}

nE é o número de elementos de cada partição, ex: $(2,1)$ tem dois elementos;

v[i] armazena a i-ésima partição de m;

$\mathbf{v}[\mathbf{i}][\mathbf{j}]$ indica o valor do elemento que está na $j$-ésima posição da partição armazenada no vetor v[i], ex: o valor do $1^{\circ}$ elemento de $(2,1)(v[i][1])$ é 2;

$\mathbf{n P}$ indica o número de partições de $\mathrm{m}$; ex: $\mathrm{m}=3,(3),(2,1),(1,1,1)$ então $\mathrm{nP}=3$;

$\mathbf{n E}[\mathbf{i}]$ armazena o número de elementos do vetor v[i];

$\mathbf{H}[\mathbf{y 1}]$ armazena as variáveis $\mathrm{z}[\mathrm{j}, \mathrm{k}]$ de todas as partições de m;

$\mathbf{t}[\mathbf{y} 2]$ armazena as variáveis que são igualdades do tipo $(\mathrm{z}[\mathrm{j}, \mathrm{k}+1]=\mathrm{z}[\mathrm{j}, 0])$ de todas as partições de $\mathrm{m}$;

L[i] armazena apenas as variáveis da i-ésima partição de m;

$\mathbf{T}[\mathbf{i}]$ armazena apenas as variáveis que são igualdades do tipo $(\mathrm{z}[\mathrm{j}, \mathrm{k}+1]=\mathrm{z}[\mathrm{j}, 0])$ da i-ésima partição de m;

V armazena a matriz de Vandermonde com respeito ao vetor (ou lista) L[i];

U é o vetor onde estão armazenados os $h_{i}$ já calculados;

As variáveis y1 e y2 auxiliam a separar as variáveis $\mathrm{z}[\mathrm{j}, \mathrm{k}]$ de acordo com cada partição.

\section{Significado dos comandos utilizados}

partition: este comando particiona um inteiro não negativo $n$, ou seja, o resultado é uma lista com entradas inteiras onde a soma dos elementos em cada lista é $n$. A ordem dos elementos de cada lista não é considerada.

ex.: $\operatorname{partition}(3)=[[1,1,1],[1,2],[3]]$.

numbpart: este comando conta o número de partições de um inteiro não negativo $n$. 
ex.: numbpart(3)=3.

decodepart: Dado um inteiro positivo $n$ e um inteiro positivo $m$ entre 1 e numbpart(n), a função decodepart(m, n) computa e retorna a única partição representada por este inteiro positivo. ex.: $\operatorname{decodepart}(3,1)=[1,1,1]$.

ArrayNumElems(A): Esta função retorna o número de elementos armazenados em um Array A.

seq: Esta função é usada para construir uma sequência de valores.

ex.: $\operatorname{seq}(f(i), i=m . . n)$ gera a sequência: $f(m), f(m+1), \ldots, f(n)$.

nops: esta função retorna o número de operandos de uma expressão.

ex.: $\mathrm{u}:=[1,4,9]$ então $\operatorname{nops}(\mathrm{u})=3$.

vandermonde(L): esta função retorna a matriz de Vandermonde formados com elementos da lista L.

subs: este comando substitui uma subexpressão em outra expressão.

ex.: $\operatorname{subs}\left(x=2, x^{2}+1\right)=5$.

Para usar o algoritmo entre com o seguinte comando:

$$
\operatorname{ideais}(g, m, m 1, m 2)
$$

onde, $g$ é o germe dado, $m$ um inteiro positivo $(m \leq n)$ e $m 1, m 2(1 \leq m 1 \leq m 2 \leq n u m b p a r t(m))$ são inteiros positivos que definem o intervalo de partições do qual queremos calcular os ideais. ex.: na configuração ideais $(g, 3,1,3)$ são calculados os ideais para todas partições de $m=3$. Mas na configuração ideais $(g, 3,1,2)$ são calculados os ideais para somente as duas primeiras partições de $m=3$ que são:[1,1,1] e [1,2]. Quando se tem $m 1=m 2$ apenas a $m 1$-ésima partição é usada no cálculo dos ideais.

A seguir, aplicamos este algoritmo nos exemplos da introdução.

1. Seja $f: \mathbb{C}^{2} \rightarrow \mathbb{C}^{2}$ dada por $f(x, z)=\left(x, z^{4}+x z-z^{2}\right)$.

Para $m=2$, façamos: ideais $\left(z^{4}+x z-z^{2}, 2,1,2\right)$

$-g=z^{4}+x z-z^{2}$

$-m=2$

$-m 1=1$ 
$-m 2=2$

- Partições $=[[1,1],[2]]$

Para a partição $\mathscr{P}=[1,1]$, cálculo dos pontos duplos, temos $\mathscr{J}^{2}(f, \mathscr{P})=\left\langle h_{1}, h_{2}, h_{3}\right\rangle$, com $h_{1}=2 z_{2}^{2} z_{1}+2 z_{2} z_{1}^{2}+x, h_{2}=-z_{2}^{2}-4 z_{1} z_{2}-1-z_{1}^{2}$ e $h_{3}=2 z_{2}+2 z_{1}$.

Para a partição $\mathscr{P}=[2]$, cálculo dos pontos cuspidais, temos $\mathscr{J}^{1}(f, \mathscr{P})=\left\langle h_{1}, h_{2}\right\rangle$, com $h_{1}=-8 z_{1}^{3}+x, h_{2}=6 z_{1}^{2}-1$.

Para $m=1$, façamos: ideais $\left(z^{4}+x z-z^{2}, 1,1,1\right)$

$-g=z^{4}+x z-z^{2}$

$-m=1$

$-m 1=1$

$-m 2=1$

- Partição = [1]

Para a partição $\mathscr{P}=[1]$, cálculo do conjunto singular, temos $\mathscr{J}^{1}(f, \mathscr{P})=\left\langle 4 z_{1}^{3}-2 z_{1}+x\right\rangle$.

2. Seja $f: \mathbb{C}^{3} \rightarrow \mathbb{C}^{3}$ dada por $f(x, y, z)=\left(x, y, z^{5}+x z^{2}+y z-z^{3}\right)$.

Para $m=3$, façamos: ideais $\left(z^{5}+x z^{2}+y z-z^{3}, 3,1,3\right)$

$-g=z^{5}+x z^{2}+y z-z^{3}$

$-m=3$

$-m 1=1$

$-m 2=3$

- Partições $=[[1,1,1],[1,2],[3]]$

Para a partição $\mathscr{P}=[1,1,1]$, cálculo dos pontos triplos, temos $\mathscr{J}^{3}(f, \mathscr{P})=\left\langle h_{1}, h_{2}, h_{3}, h_{4}, h_{5}\right\rangle$ $\operatorname{com} h_{1}=y, h_{2}=x, h_{3}=-1, h_{4}=0$ e $h_{5}=1$.

Observação: Como temos um dos $h_{i}=1$, isto mostra que este exemplo não tem pontos triplos.

Para a partição $\mathscr{P}=[1,2]$, cruzamento do eixo cuspidal com o plano, temos

$$
\begin{gathered}
\mathscr{J}^{2}(f, \mathscr{P})=\left\langle h_{1}, h_{2}, h_{3}, h_{4}\right\rangle, \text { com } \\
h_{1}=-3 z_{2}^{2} z_{1}^{2}-2 z_{2}^{3} z_{1}+y
\end{gathered}
$$




$$
\begin{gathered}
h_{2}=6 z_{2}^{2} z_{1}+z_{2}^{3}+3 z_{1}^{2} z_{2}+x \\
h_{3}=-6 z_{1} z_{2}-z_{1}^{2}-3 z_{2}^{2}-1 \\
h_{4}=2 z_{1}+3 z_{2},
\end{gathered}
$$

Para a partição $\mathscr{P}=[3]$, cálculo dos pontos rabo de andorinha, temos

$$
\begin{gathered}
\mathscr{J}^{1}(f, \mathscr{P})=\left\langle h_{1}, h_{2}, h_{3}\right\rangle, \text { com } \\
h_{1}=15 z_{1}^{4}+y \\
h_{2}=-20 z_{1}^{3}+x \\
h_{3}=10 z_{1}^{2}-1
\end{gathered}
$$

Para $m=2$, façamos: ideais $\left(z^{5}+x z^{2}+y z-z^{3}, 2,1,2\right)$

$-g=z^{5}+x z^{2}+y z-z^{3}$

$-m=2$

$-m 1=1$

$-m 2=2$

- Partições $=[[1,1],[2]$

Para a partição $\mathscr{P}=[1,1]$, cálculo da curva de pontos duplos, temos

$$
\begin{gathered}
\mathscr{J}^{2}(f, \mathscr{P})=\left\langle h_{1}, h_{2}, h_{3}\right\rangle, \text { com } \\
h_{1}=7 z_{1}^{2} z_{2}^{2}+4 z_{1}^{3} z_{2}+4 z_{1} z_{2}^{3}+y \\
h_{2}=-8 z_{2} z_{1}^{2}-2 z_{1}^{3}-8 z_{1} z_{2}^{2}-2 z_{2}^{3}+x \\
h_{3}=3 z_{1}^{2}+4 z_{1} z_{2}+3 z_{2}^{2}-1 .
\end{gathered}
$$

Para a partição $\mathscr{P}=[2]$, cálculo da curva cuspidal, temos $\mathscr{J}^{1}(f, \mathscr{P})=\left\langle h_{1}, h_{2}\right\rangle$ com

$$
h_{1}=-15 z_{1}^{4}+3 z_{1}^{2}+y
$$




$$
h_{2}=10 z_{1}^{3}-3 z_{1}+x
$$

Para $m=1$, façamos: ideais $\left(z^{5}+x z^{2}+y z-z^{3}, 1,1,1\right)$

$-g=z^{5}+x z^{2}+y z-z^{3}$

- $m=1$

$-m 1=1$

$-m 2=1$

- Partição = [1]

Para a partição $\mathscr{P}=[1]$, cálculo do conjunto singular, temos $\mathscr{J}^{1}(f, \mathscr{P})=\left\langle 5 z_{1}^{4}-3 z_{1}^{2}+\right.$ $\left.2 z_{1} x+y\right\rangle$. 


\section{Capítulo}

\section{3}

\section{Multiplicidades de 0-esquemas de germes}

\section{quase homogêneos em $\mathscr{O}(n, n)$}

Neste capítulo são descritas fórmulas para o cálculo das multiplicidades associadas aos zeroesquemas que ocorrem no discriminante do germe. O principal objetivo é investigar a seguinte questão: Dado um germe de aplicação $f:\left(\mathbb{C}^{n}, 0\right) \rightarrow\left(\mathbb{C}^{n}, 0\right), \mathscr{A}$-finito e uma partição $\mathscr{P}$ de $n$, quantas singularidades de tipo $A_{p}$ existem em uma estabilização de $f$. Denotamos este número por $\# A_{p}=\# A_{p}(f)$

Estes resultados foram descritos em [2].

Para germes de aplicações de coposto 1, escolhemos um sistema de coordenadas conveniente, e escrevemos

$$
\begin{aligned}
f: \mathbb{C}^{n-1} \times \mathbb{C} & \rightarrow \mathbb{C}^{n-1} \times \mathbb{C} \\
(x, z) & \mapsto(x, g(x, z))
\end{aligned}
$$

Definição 3.1. Um germe de aplicação analítica $f:\left(\mathbb{C}^{n}, 0\right) \rightarrow\left(\mathbb{C}^{p}, 0\right), f=\left(f_{1}, \ldots, f_{p}\right)$ é quase homogêneo, de tipo $\left(\omega_{1}, \ldots, \omega_{n} ; d_{1}, \ldots, d_{p}\right)$ se existem inteiros positivos $\omega_{1}, \omega_{2}, \ldots, \omega_{n}$ (os pesos) e inteiros positivos $d_{1}, d_{2}, \ldots, d_{p}$, (os graus), tal que para todo $x \in \mathbb{C}^{n}, \lambda \in \mathbb{C}$.

$$
f_{i}\left(\lambda^{\omega_{1}} x_{1}, \lambda^{\omega_{2}} x_{2}, \ldots, \lambda^{\omega_{n}} x_{n}\right)=\lambda^{d_{i}} f_{i}(x)
$$

Equivalentemente, para todo monômio $x_{1}^{\alpha_{1}} x_{2}^{\alpha_{2}} \ldots x_{n}^{\alpha_{n}}$ que aparece na expansão de Taylor da $i$-ésima componente $f_{i}$ de $f$, temos $\sum_{j=1}^{n} \omega_{j} \alpha_{j}=d_{i}$. 
Definição 3.2. Um germe $f: \mathbb{C}^{n} \rightarrow \mathbb{C}^{p}$ é semi quase homogêneo de tipo $\left(\omega_{1}, \ldots, \omega_{n} ; d_{1}, \ldots, d_{p}\right)$ se $f=\left(f_{1}, f_{2}, \ldots, f_{p}\right)$ se escreve como

$$
f=\left(f_{1}, f_{2}, \ldots, f_{p}\right)=\left(f_{1}^{0}+f_{1}^{1}+\cdots, f_{2}^{0}+f_{2}^{1}+\cdots, \ldots, f_{p}^{0}+f_{p}^{1}+\cdots\right)
$$

com cada $f_{i}=f_{i}^{0}+f_{i}^{1}+\cdots$ e o germe $f^{0}=\left(f_{1}^{0}, f_{2}^{0}, \ldots, f_{p}^{0}\right)$ é finitamente determinado e quase homogêneo de tipo $\left(\omega_{1}, \ldots, \omega_{n} ; d_{1}, \ldots, d_{p}\right)$ e cada $f_{i}^{j}$ com $j>0$ é quase homogêneo de tipo $\left(\omega_{1}, \ldots, \omega_{n}, d_{1}^{j}, \ldots, d_{p}^{j}\right) \operatorname{com} d_{i}^{j+1}>d_{i}^{j}, i=1, \ldots, p, j=1, \ldots, n$.

O teorema a seguir nos dá uma resposta para a questão acima, para o caso de germes quase homogêneos de coposto 1. Para isto fixamos a notação.

Seja $\mathscr{P}=\left(r_{1}, \ldots, r_{\ell}\right)$ uma partição de $n$, com $r_{1} \geq r_{2} \geq \ldots \geq r_{\ell}, \ell$ igual ao comprimento de $\mathscr{P}$. Defina $N(\mathscr{P})$ a ordem do subgrupo de $S_{\ell}$ que fixa $\mathscr{P}$. Aqui $S_{\ell}$ age sobre $\mathbb{C}^{\ell}$ por permutação em suas coordenadas. Por exemplo: Se $\mathscr{P}=(4,4,4,3,3,1,1) \Rightarrow N(\mathscr{P})=3 ! 2 ! 2 !=24$.

Teorema 3.3 ([2], Teorema 1). Seja $f:\left(\mathbb{C}^{n}, 0\right) \rightarrow\left(\mathbb{C}^{n}, 0\right), f\left(x_{1}, \ldots, x_{n-1}, z\right)=\left(x_{1}, \ldots, x_{n-1}, g(x, z)\right)$ germe de aplicação $\mathscr{A}$-finito, com g quase homogêneo de tipo $\left(\omega_{1}, \ldots, \omega_{n-1}, \omega_{0} ; d\right)$. Para qualquer estabi-lização de f e qualquer partição $\mathscr{P}$ de $n$

$$
\# A_{p}=\frac{\omega_{0}^{n-1}}{N(\mathscr{P}) \omega} \prod_{j=1}^{n+\ell-1}\left(\frac{d}{\omega_{0}}-j\right)
$$

$\operatorname{com} \omega=\prod_{i=1}^{n-1} \omega_{i}$

A demonstração do teorema anterior decorre das proposições 3.4 ,3.5, 3.6 e 3.8 seguintes.

Seja $F:\left(\mathbb{C}^{d} \times \mathbb{C}^{n-1} \times \mathbb{C},(0,0,0)\right) \rightarrow\left(\mathbb{C}^{d} \times \mathbb{C}^{n-1} \times \mathbb{C},(0,0,0)\right)$ um desdobramento $\mathscr{A}_{e}$-versal de $f$, a $d$-parâmetros, então temos, $F(u, x, z)=\left(u, f_{u}(x, z)\right)$.

Para Cada partição $\mathscr{P}=\left(r_{1}, \ldots, r_{\ell}\right)$ de $n$, vamos considerar o subesquema $D^{\ell}(F, \mathscr{P})$ em $\mathbb{C}^{d} \times \mathbb{C}^{n-1} \times \mathbb{C}^{\ell}$ como definido na igualdade 2.3 .

Seja $\pi=\pi(\mathscr{P}): D^{\ell}(F, \mathscr{P}) \rightarrow \mathbb{C}^{d}$ a restrição ao conjunto $D^{\ell}(F, \mathscr{P})$ da projeção cartesiana $\mathbb{C}^{d} \times \mathbb{C}^{n-1} \times \mathbb{C}^{\ell} \rightarrow \mathbb{C}^{d}$. Para um ponto genérico $u \in \mathbb{C}^{d}$, a fibra $\pi^{-1}(u)$ consiste dos pontos múltiplos em que $f$ tem um $\mathscr{A}_{p}$ multigerme. 
Proposição 3.4 ([2], Proposição 2). Se $\mathscr{P}=\left(r_{1}, \ldots, r_{\ell}\right)$ é uma partição de $n$, então

$$
\# A_{p}=\frac{1}{N(\mathscr{P})} \operatorname{grau}(\pi(\mathscr{P}))
$$

Demonstração: Seja $w=\left(u, x, z_{1}, \ldots, z_{\ell}\right) \in D^{\ell}(F, \mathscr{P})$ e $\sigma \in S^{\ell}$, onde $S^{\ell}$ permuta as últimas $\ell$-coordenadas $z_{i}$ de $w$. Então $w^{\sigma}=\left(u, x, z_{\sigma(1)}, \ldots, z_{\sigma(\ell)}\right)$. Daí, $w^{\sigma} \in D^{\ell}(F, \mathscr{P})$ se e somente se $r_{\sigma(j)}=r_{j}$. Para cada $j=1, \ldots, \ell$ existem $N(\mathscr{P})$ tais $\sigma$. Os pontos $w$ e $w^{\sigma}$ são distintos, mas os conjuntos correspondentes são os mesmos e são estes os conjuntos que são contados em $\# A_{p}$.

Lembremos que $\pi^{-1}(u) \in D^{\ell}(F, \mathscr{P})$ e o $\operatorname{grau}(\pi(\mathscr{P}))$ é o número de pré-imagens de $\pi(\mathscr{P})$. Então o que nos interessa agora é o $\operatorname{grau}(\pi(\mathscr{P}))$. Sejam $\mathscr{J}_{\Delta}^{\ell}(F, \mathscr{P})$ o ideal que define $D^{\ell}(F, \mathscr{P})$ e $\mathscr{J}_{u}^{\ell}(F, \mathscr{P})=\frac{\mathscr{J}_{\Delta}^{\ell}(F, \mathscr{P})+<u_{1}, \ldots, u_{d}>}{<u_{1}, \ldots, u_{d}>} \subset \mathscr{O}_{\mathbb{C}^{n-1} \times \mathbb{C}^{\ell}}$ correspondendo à intersecção de $D^{\ell}(F, \mathscr{P})$ com $\{0\} \times \mathbb{C}^{n-1} \times \mathbb{C}^{\ell}$, assim o ideal $\mathscr{J}_{u}^{\ell}(F, \mathscr{P})$ define a fibra $\pi(\mathscr{P})^{-1}(0)$.

Proposição 3.5 ([2], Proposição 4). $\mathscr{J}_{u}^{\ell}(F, \mathscr{P})$ é interseção completa 0 -dimensional, e portanto o grau de $\pi$ coincide com $\operatorname{dim}_{\mathbb{C}} \frac{\mathscr{O}_{\mathbb{C}^{n-1} \times \mathbb{C}^{\ell}}}{\mathscr{J}_{u}^{\ell}(F, \mathscr{P})}$.

Demonstração: Seguindo os passos da demonstração da proposição 2.8 item (ii), $\mathscr{J}_{\Delta}^{\ell}(F, \mathscr{P})$ é interseção completa $d$-dimensional. Como,

$$
\mathscr{J}_{u}^{\ell}(F, \mathscr{P})=\frac{\mathscr{J}_{\Delta}^{\ell}(F, \mathscr{P})+<u>}{<u>}
$$

com $\langle u\rangle=\left\langle u_{1}, \ldots, u_{d}\right\rangle$, segue que $\mathscr{J}_{u}^{\ell}(F, \mathscr{P})$ é interseção completa 0 -dimensional. E sendo $\mathscr{J}_{u}^{\ell}(F, \mathscr{P})$ uma interseção completa então é Cohen-Macaulay e portanto o grau de $\pi(\mathscr{P})$ coincide com $\operatorname{dim}_{\mathbb{C}} \frac{\mathscr{O}_{\mathbb{C}^{n-1} \times \mathbb{C}^{\ell}}}{\mathscr{J}_{u}^{\ell}(F, \mathscr{P})}$.

Proposição 3.6 ([2], Proposição 3). $D^{\ell}(F, \mathscr{P})$ é suave de dimensão d, e $\pi(\mathscr{P}): D^{\ell}(F, \mathscr{P}) \rightarrow \mathbb{C}^{d}$ é finito a um e plana.

Demonstração: Mostremos que $D^{\ell}(F, \mathscr{P})$ é suave de dimensão $d$. Como $F$ é uma deformação

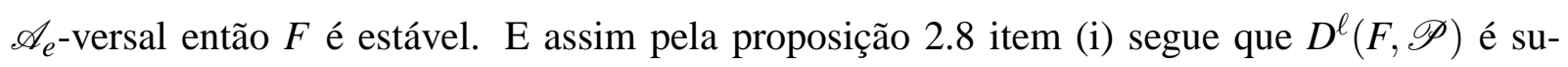
ave $d$-dimensional. Como $f$ é finitamente $\mathscr{A}$-determinado e $A_{p}$ é um multigerme 0 -estável, então pelo critério geométrico de Mather-Gaffney a fibra sobre zero ou é a origem ou é vazia pois $\pi(\mathscr{P})^{-1}(0)=V\left(\mathscr{J}_{u}^{\ell}(F, \mathscr{P})\right)$ e assim $\pi(\mathscr{P})$ é finito a um. Além do mais, o ideal $\mathscr{J}_{u}^{\ell}(F, \mathscr{P})$ definindo a fibra de $\pi$ sobre zero é obtido como a especialização de $\mathscr{J}^{\ell}(f, \mathscr{P})$ para 
a diagonal principal $\Delta_{0}$, cujos geradores são $z_{i}^{k}-z_{0}^{k}, i=1, \cdots, r_{k} ; k=1, \cdots, \ell$. Os geradores $\left\langle\frac{\partial g}{\partial z}\left(x, z^{1}\right), \cdots, \frac{\partial^{r_{1}} g}{\partial z^{r_{1}}}\left(x, z^{1}\right), \cdots, \frac{\partial g}{\partial z}\left(x, z^{\ell}\right), \cdots, \frac{\partial^{r} \ell g}{\partial z^{r}}\left(x, z^{\ell}\right)\right\rangle$ que aparecem do lema 2.1 formam uma sequência regular em $\mathbb{C}^{n+\ell-1}$. Então por [[9], pág.112] a projeção $\pi(\mathscr{P}): D^{\ell}(F, \mathscr{P}) \rightarrow \mathbb{C}^{d}$ é plana.

Observação 3.7. $\operatorname{grau}\left(h_{i}\right)=d-i \omega_{0}$. De fato, cada $h_{i}$ é da seguinte forma:

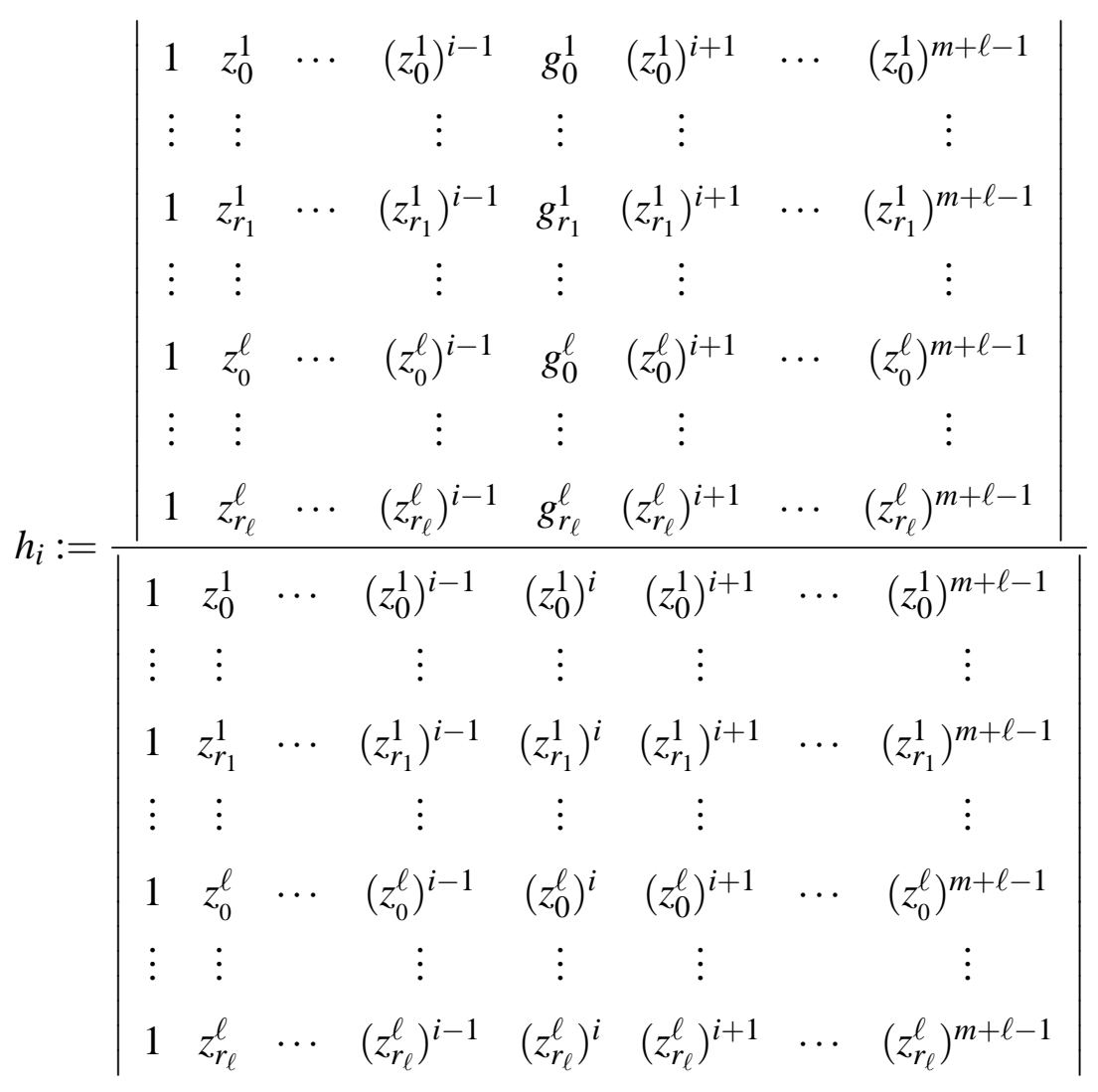

Aplicamos a regra de Sarrus para calcular o determinante acima, onde fixamos a coluna $(i+1)$ de ambas as matrizes. Então,

$$
h_{i}=\frac{(-1)^{1+(i+1)} g_{0}^{1} A_{1,(i+1)}+\ldots+(-1)^{m+\ell+(i+1)} g_{r_{\ell}}^{\ell} A_{m+\ell,(i+1)}}{(-1)^{1+(i+1)}\left(z_{0}^{1}\right)^{i} A_{1,(i+1)}+\ldots+(-1)^{m+\ell+(i+1)}\left(z_{r_{\ell}}^{\ell}\right)^{i} A_{m+\ell,(i+1)}}
$$

$A_{k,(i+1)}$ é obtido da matriz acima excluindo a linha $k$ e a coluna $(i+1), k=1, \ldots, m+\ell, i=$ $1, \ldots, m+\ell-1$ e $g_{i}^{k}:=g\left(x, z_{i}^{k}\right)$. Como os pesos das variáveis $z_{r_{j}}^{j}$, são todos iguais a $\omega_{0}$, segue que o $\operatorname{grau}\left(A_{k,(i+1)}\right)$ é o mesmo para todo $k=1, \ldots, m+\ell, i=1, \ldots, m+\ell-1$. Logo para 
calcular o grau de $h_{i}$ basta considerar uma das parcelas do numerador e uma do denominador da igualdade 3.1 acima. Portanto,

$$
\operatorname{grau}\left(h_{i}\right)=\operatorname{grau}\left(\frac{g_{0}^{1} A_{1,(i+1)}}{\left(z_{0}^{1}\right)^{i} A_{1,(i+1)}}\right)=\operatorname{grau}\left(\frac{g_{0}^{1}}{\left(z_{0}^{1}\right)^{i}}\right)=d-i \omega_{0}
$$

Proposição 3.8 ([2], Proposição 5). Se $f(x, z)=(x, g(x, z))$ com g quase homogêneo de tipo $\left(\omega_{1}, \ldots, \omega_{n-1}, \omega_{0} ; d\right)$, a projeção $\pi(\mathscr{P}): D^{\ell}(F, \mathscr{P}) \rightarrow \mathbb{C}^{d}$ tem grau

$$
d(\mathscr{P})=\frac{1}{\omega_{0}^{\ell} \omega} \prod_{j=1}^{n+\ell-1}\left(d-j \omega_{0}\right)
$$

Demonstração: Aplicamos o teorema de Bezout ao ideal $\mathscr{J}^{\ell}(f, \mathscr{P})+\mathscr{I}_{\Delta(\mathscr{P})}$ para o cálculo de seu grau. Para isto precisamos dos graus de cada componente deste ideal e como $f$ é quase homogêneo também serão usados os pesos nas variáves $(x, \mathbf{z}) \in \mathbb{C}^{n-1} \times \mathbb{C}^{n+\ell}$ onde $(x, \mathbf{z})$ é da forma 2.4. Temos

$$
\begin{aligned}
V\left(\mathscr{J}^{\ell}(f, \mathscr{P})+\mathscr{I}_{\Delta(\mathscr{P})}\right) & =V\left(\mathscr{J}^{\ell}(f, \mathscr{P}), \mathscr{I}_{\Delta(\mathscr{P})}\right) \\
& =V\left(h_{1}, \ldots, h_{m+\ell-1}, z_{1}^{1}-z_{0}^{1}, \ldots, z_{r_{1}}^{1}-z_{0}^{1}, \ldots, z_{1}^{\ell}-z_{0}^{\ell}, \ldots, z_{r_{\ell}}^{\ell}-z_{0}^{\ell}\right) .
\end{aligned}
$$

Os pesos nas variáveis $x_{i}$ são iguais a $\omega_{i}, i=1, \ldots, n-1$ e o peso em $\mathbf{z}$ é $\omega_{0}$. Para o cálculo do grau de cada componente $z_{r_{i}}^{i}-z_{0}^{i}$ de $\mathscr{I}_{\Delta(\mathscr{P})}$, considere as aplicações:

$$
\begin{gathered}
\psi_{i, j}: \mathbb{C}^{n-1} \times \mathbb{C}^{n+\ell} \rightarrow \mathbb{C} \\
(x, \mathbf{z}) \mapsto z_{r_{i}}^{i}-z_{0}^{i}
\end{gathered}
$$

com $i=1, \ldots, \ell, j=0, \ldots, r_{i}, \mathrm{e}(x, \mathbf{z})$ como na igualdade 2.4. $\psi_{i, j}$ só depende das últimas $n+\ell$ variáveis, onde os pesos são todos iguais a $\omega_{0}$. Vemos que cada $\psi_{i, j}$ tem grau igual a $\omega_{0}$, como temos $n$ aplicacões $\psi_{i, j}$, o produto dos graus é $\left(\omega_{0}\right)^{n}$. Assim,

$$
\operatorname{grau}\left(\mathscr{J}^{\ell}(f, \mathscr{P}), \mathscr{I}_{\Delta(\mathscr{P})}\right)=\frac{\prod_{j=1}^{n+\ell-1}\left(d-j \omega_{0}\right)\left(\omega_{0}\right)^{n}}{\omega\left(\omega_{0}\right)^{n+\ell}}=\frac{1}{\omega_{0}^{\ell} \omega} \prod_{j=1}^{n+\ell-1}\left(d-j \omega_{0}\right)
$$

com, $\omega=\omega_{1} \omega_{2} \ldots \omega_{n-1}$.

A seguir calculamos as multiplicidades dos tipos 0-estáveis ou 0-esquemas dos exemplos 
mostrados na introdução.

1. $f(x, z)=\left(x, z^{4}+x z\right)$. Neste caso $g(x, z)=z^{4}+x z$, que é quase homogêneo de tipo $(3,1 ; 4)$.

Para calcular a multiplicidade dos pontos de cúspide (pontos $-A_{2}$ ), a partição de $n=2$ a ser considerada é $\mathscr{P}=(2), N(\mathscr{P})=1$. Para calcular a multiplicidade dos pontos duplos (pontos $-A_{1,1}$ ), a partição de $n=2$ a ser considerada é $\mathscr{P}=(1,1), N(\mathscr{P})=2$ e assim pelo teorema 3.3, temos:

$$
\begin{aligned}
& \# A_{2}=\frac{\omega_{0}^{n-1}}{N(\mathscr{P}) \omega} \prod_{j=1}^{n+\ell-1}\left(\frac{d}{\omega_{0}}-j\right)=\frac{1}{3} \prod_{j=1}^{2}(4-j)=2 \\
& \# A_{1,1}=\frac{\omega_{0}^{n-1}}{N(\mathscr{P}) \omega} \prod_{j=1}^{n+\ell-1}\left(\frac{d}{\omega_{0}}-j\right)=\frac{1}{6} \prod_{j=1}^{3}(4-j)=1
\end{aligned}
$$

Portanto, o número de cúspides de $f(x, z)=\left(x, z^{4}+x z\right)$ é 2 e o de pontos duplos é 1 .

2. $f(x, y, z)=\left(x, y, z^{5}+x z^{2}+y z\right)$. Neste caso $g(x, y, z)=z^{5}+x z^{2}+y z$, que é quase homogêneo de tipo $(3,4,1 ; 5)$.

Para calcular a multiplicidade dos pontos rabo de andorinha (pontos $-A_{3}$ ), a partição de $n=3$ a ser considerada é $\mathscr{P}=(3), N(\mathscr{P})=1$. Para calcular a multiplicidade dos pontos dobracúspide (pontos $-A_{1,2}$ ), a partição de $n=3$ a ser considerada é $\mathscr{P}=(1,2), N(\mathscr{P})=1$ e assim pelo teorema 3.3, temos:

$$
\begin{gathered}
\# A_{3}=\frac{\omega_{0}^{n-1}}{N(\mathscr{P}) \omega} \prod_{j=1}^{n+\ell-1}\left(\frac{d}{\omega_{0}}-j\right)=\frac{1}{12} \prod_{j=1}^{3}(5-j)=2 \\
\# A_{1,2}=\frac{\omega_{0}^{n-1}}{N(\mathscr{P}) \omega} \prod_{j=1}^{n+\ell-1}\left(\frac{d}{\omega_{0}}-j\right)=\frac{1}{12} \prod_{j=1}^{4}(5-j)=2
\end{gathered}
$$

Já foi observado que os pontos triplos não ocorrem neste exemplo.

Portanto, o número de rabos de andorinhas de $f(x, y, z)=\left(x, y, z^{5}+x z^{2}+y z\right)$ é 2 e o de dobra-cúspide também é 2. 


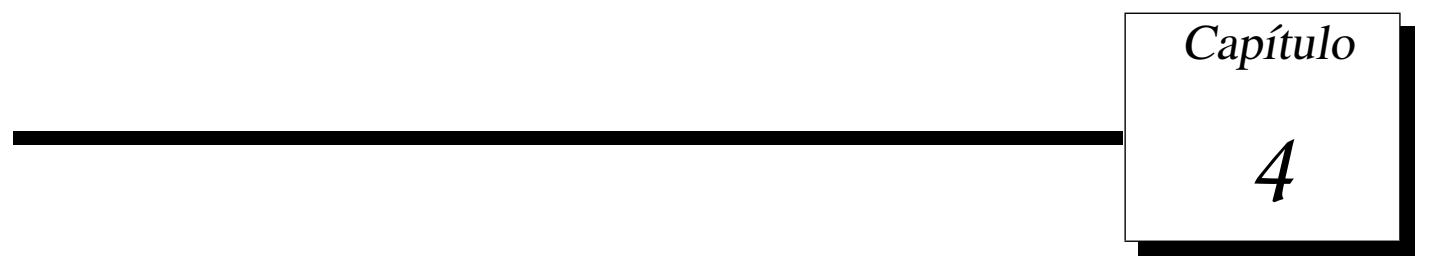

\section{Multiplicidades polares do discriminante em}

\section{germes quase homogêneos em $\mathscr{O}(3,3)$}

A determinação das multiplicidades polares definidas sobre os tipos estáveis é essencial para a obtenção da Whitney equisingularidade de famílias de aplicações. Usando multiplicidades polares e os Lemas de isotopia de Thom, Gaffney mostrou o importante teorema.

Teorema 4.1. Suponha que $F: \mathbb{C} \times \mathbb{C}^{n} \rightarrow \mathbb{C} \times \mathbb{C}^{p}$ seja um desdobramento excelente de $f \in$ $\mathscr{O}(n, p)$. Então $F$ é Whitney equisingular se e somente se as multiplicidades polares de todos os tipos estáveis definidas nos conjuntos:

1. conjunto singular $\Sigma\left(f_{t}\right)$;

2. discriminante $\Delta\left(f_{t}\right)=f_{t}\left(\Sigma\left(f_{t}\right)\right)$;

3. $X\left(f_{t}\right)=\overline{\left.\left(f_{t}^{-1}\left(\Delta\left(f_{t}\right)\right)-\Sigma f_{t}\right)\right)}$, são constantes na origem para todo $t$.

Neste capítulo são calculadas as multiplicidades polares definidas sobre os tipos estáveis no discriminante, para germes $f:\left(\mathbb{C}^{3}, 0\right) \rightarrow\left(\mathbb{C}^{3}, 0\right)$ finitamente determinados quase homogêneos de coposto 1 em função dos pesos e graus de $f$. Estes resultados são descritos em [4]

\subsection{Multiplicidades polares no discriminante}

Inicialmente são descritas as variedades polares. As multiplicidades associadas a estas variedades, são chamadas por Tessier em [13] de multiplicidades polares.

Seja $X \subset \mathbb{C}^{n}$ uma variedade qualquer de dimensão $d$. Por $P_{k}(X)$ é denotada a $k$-variedade polar absoluta de $X, \operatorname{com} k=0, \ldots, d-1$, cuja construção é feita a seguir: 
Seja $p_{k}: \mathbb{C}^{n} \rightarrow \mathbb{C}^{d-k+1}$ uma projeção linear genérica, definimos $P_{k}(X)$ como sendo o fecho do conjunto singular de $p_{k}$ restrito ao conjunto $X-\sin g(X)$. Ou seja,

$$
P_{k}(X)=\overline{\Sigma\left(p_{k} \mid X^{0}\right)}
$$

com $X^{0}=X-\operatorname{sing}(X)$, onde $\operatorname{sing}(X)$ é a parte singular de $X$.

O invariante principal de $P_{k}(X)$ é sua multiplicidade, chamada multiplicidade polar absoluta a qual é denotada por $m_{0}\left(P_{k}(X)\right)$ ou $m_{k}(X)$.

Multiplicidades polares absolutas são definidas se a dimensão de $X$ é maior ou igual a 1 .

As multiplicidades $m_{d}(X)$ não podem ser definidas da mesma forma como as outras $m_{k}(X)$, $0 \leq k \leq d-1$, pois as singularidades de $p_{d} \mid X$ são pontos isolados. Por isso Gaffney em [[10], p.195] define um novo invariante da seguinte maneira:

Tome um desdobramento versal $F:\left(\mathbb{C}^{n},(0,0)\right) \times\left(\mathbb{C}^{s},(0,0)\right) \rightarrow \mathbb{C}^{p} \times \mathbb{C}^{s}$ de $f$, especifique um tipo de singularidade estável ou estrato $\mathscr{Q}(f)$ na fonte ou na meta tal que a $\operatorname{dim} \overline{\mathscr{Q}(f)} \geq 1$. Selecione $D_{1}$ um subespaço linear de $\left(\mathbb{C}^{n}, 0\right)$ (ou $\left(\mathbb{C}^{p}, 0\right)$ se estiver trabalhando na meta) de codimensão 1 e considere $P_{d}(\mathscr{Q}(F))$ a variedade polar de $\mathscr{Q}(F)$ através da projeção $\left(p, \pi_{s}\right)$ : $\mathbb{C}^{n} \times \mathbb{C}^{s} \rightarrow \mathbb{C} \times \mathbb{C}^{s}$ onde $d=\operatorname{dim} \overline{\mathscr{Q}(F)}-s$.

A d-ésima multiplicidade estável de $f$ de tipo $\mathscr{Q}(f)$ denotada por $m_{d}(\mathscr{Q}(f))$ é a multiplicidade de $m_{s} \mathscr{O}_{\overline{\mathscr{Q}(F)},(0,0)}$ em $\mathscr{O}_{\overline{\mathscr{Q}(F)},(0,0)}$.

No caso em que $X$ é uma I.C.I.S., Gaffney em [[11], p.211] dá uma fórmula para o cálculo da multiplicidade $m_{d}(X)$

Teorema 4.2. Seja $f:\left(\mathbb{C}^{n}, 0\right) \rightarrow\left(\mathbb{C}^{n-d}, 0\right), f^{-1}(0)=X^{d}, X$ uma I.C.I.S. de dimensão d e $p$ : $\mathbb{C}^{n} \rightarrow \mathbb{C}$ é uma projeção linear genérica, então

$$
m_{d}(X)=\operatorname{dim} \frac{\mathscr{O}_{X}}{J(p, f)}
$$

Demonstração: Lema 5.8 de [11].

A seguir descrevemos os resultados de [4] que são essenciais para a obtenção das fórmulas para as multiplicidades polares.

Estes resultados descrevem as relações entre as multiplicidades polares dos tipos estáveis no discriminante e os invariantes 0-estáveis associados ao germe. 
De acordo com o teorema 2.11 item $i$ ), no caso de germes de $\mathbb{C}^{3}$ em $\mathbb{C}^{3}$, os tipos estáveis na meta, são os seguintes:

(i) O discriminante $\Delta(f)$ de $f$

(ii) A imagem da curva cuspidal $f\left(\Sigma^{1,1}(f)\right)$

(iii) A imagem da curva de pontos duplos $f\left(D_{1}^{2}(f \mid \Sigma(f))\right)$

Teorema 4.3 ([4], Teorema 3.1). Seja $f \in \mathscr{O}(3,3)$ um germe finitamente determinado e $p: \mathbb{C}^{3} \rightarrow$ $\mathbb{C}^{2}$ uma projeção linear genérica. Então

$$
\mu(p \circ f)+\delta(f)-1=m_{0}(\Delta(f)) .
$$

onde, $\delta(f)=\operatorname{dim}_{\mathbb{C}} \frac{\mathscr{O}_{n}}{f^{*}\left(m_{n}\right) \mathscr{O}_{n}}$ é o grau de $f$.

Teorema 4.4 ([4], Teorema 3.3). Suponhamos que $f \in \mathscr{O}(3,3)$ é um germe finitamente determinado. Então

$$
\left.m_{2}(\Delta(f))-m_{1}(\Delta(f))+m_{0}(\Delta(f))=\mu(\Sigma(f))\right)+1
$$

Teorema 4.5 ([4], Teorema 3.5). Seja $f \in \mathscr{O}(3,3)$ germe finitamente determinado de coposto 1. Então

$$
2 m_{0}\left(f\left(D_{1}^{2}(f / \Sigma(f))\right)\right)-2 m_{1}\left(f\left(D_{1}^{2}(f / \Sigma(f))\right)\right)+\mu\left(D_{1}^{2}(f / \Sigma(f))\right)=3 \# A_{1} A_{2}+3 \# A_{3}+6 \# A_{1}^{3}+1
$$

Teorema 4.6 ([4], Teorema 3.6). Seja $f \in \mathscr{O}(3,3)$ germe finitamente determinado de coposto 1. Então

$$
\left.m_{0}\left(f\left(\Sigma^{1,1}(f)\right)\right)-m_{1}\left(f\left(\Sigma^{1,1}(f)\right)\right)=1-\mu\left(\Sigma^{1,1}(f)\right)\right)+\# A_{3}
$$

O resultados abaixo são uteis na determinação destas fórmulas.

Proposição 4.7 ([15]). Seja $f \in \mathscr{O}(3,3)$ germe finitamente determinado de coposto 1 . Então

$$
\mu\left(D_{1}^{2}(f / \Sigma(f))\right)=\mu\left(D^{2}(f)\right)+2 \# A_{1} A_{2}+6 \# A_{1}^{3}
$$


Teorema 4.8 ([14], pp. 77). Seja $f:\left(\mathbb{C}^{n}, 0\right) \rightarrow\left(\mathbb{C}^{p}, 0\right), f=\left(f_{1}, \ldots, f_{p}\right)$ um germe de aplicação analítica quase homogêneo com pesos $\omega_{1}, \ldots, \omega_{n}$ e graus $d_{1}, \ldots, d_{p}$. Então são válidas as seguintes igualdades:

a) $\operatorname{Se} d_{1}=\ldots=d_{p}=d$ :

$$
\mu(f)=(-1)^{n-p+1}+(-1)^{n-p} \frac{d^{n}}{\omega_{1} \cdot \ldots \cdot \omega_{n}} \sum_{\substack{0 \leq l \leq n-p \\ 1 \leq v_{1}<\ldots<v_{l} \leq n}}(-1)^{l} \prod_{\lambda=1}^{l}\left(1-\frac{\omega_{\nu_{\lambda}}}{d}\right)
$$

b) $\operatorname{Se} d_{i} \neq d_{j}$, para $i \neq j$ :

$$
\mu(f)=\sum_{\rho=1}^{p} \prod_{v=1}^{n}\left(\frac{d_{\rho}}{\omega_{v}}-1\right) \prod_{\substack{\kappa=1 \\ \kappa \neq \rho}}^{p}\left(\frac{1}{\frac{d_{\rho}}{d_{\kappa}}-1}\right)
$$

\subsection{Fórmulas para multiplicidades polares em $\Delta(f)$}

Nosso primeiro conjunto a ser estudado é o próprio discriminante de $f, \Delta(f)$. Como este conjunto é 2-dimensional, associados a este temos 3 multiplicidades polares: $m_{0}(\Delta(f)), m_{1}(\Delta(f))$ e $m_{2}(\Delta(f))$.

Teorema 4.9 ([5], Teorema 3.1). Suponha que $f=(x, y, g(x, y, z)) \in \mathscr{O}(3,3)$ é um germe finitamente determinado de coposto 1 quase homogêneo, com pesos $\omega_{1}, \omega_{2}$ e $\omega_{3}$ nas variáveis $x, y, z$ respectivamente e dé o grau de g. Então

1. $m_{1}(\Delta(f))=\frac{\left(d-2 \omega_{3}\right)\left(d-\omega_{3}\right)}{\max \left\{\omega_{1}, \omega_{2}\right\} \cdot \omega_{3}}$

2. $m_{o}(\Delta(f))=\frac{\left(d-\omega_{3}\right)}{\omega_{3}}$

3. $m_{2}(\Delta(f))=\frac{\left(d-2 \omega_{3}\right)\left(d-\omega_{3}\right)}{\max \left\{\omega_{1}, \omega_{2}\right\} \cdot \omega_{3}}+\frac{\left(d-2 \omega_{3}\right)\left(d-\omega_{3}\right)\left(d-\omega_{1}-\omega_{2}-\omega_{3}\right)}{\omega_{1} \cdot \omega_{2} \cdot \omega_{3}}$

Demonstração do item 1. Usando a relação do Teorema 4.4 para determinar $m_{1}(\Delta(f))$, são escolhidas projeções lineares genéricas $p_{1}: \mathbb{C}^{3} \rightarrow \mathbb{C}$ e $p_{2}: \mathbb{C}^{3} \rightarrow \mathbb{C}^{2}$ tais que

$$
m_{1}(\Delta(f))=\operatorname{dim}_{\mathbb{C}} \frac{\mathscr{O}_{3}}{\left(p_{1} \circ f, J[f], J\left[p_{2} \circ f, J[f]\right]\right)}
$$


O ideal $\left(p_{1} \circ f, J[f], J\left[p_{2} \circ f, J[f]\right]\right)$ nem sempre é quase homogêneo. Mas, pelo teorema 5.1 de [14] o número de Milnor de um espaço analítico semi quase homogêneo que é I.C.I.S. é igual ao número de Milnor da parte inicial deste espaço.

Vamos verificar que o ideal $\left(p_{1} \circ f, J[f], J\left[p_{2} \circ f, J[f]\right]\right)$ é semi quase homogêneo.

Seja $p_{2}(x, y, z)=\left(a x+b y+c z, a_{1} x+a_{2} y+a_{3} z\right)$ uma projeção genérica. Então

$$
\begin{gathered}
J\left[p_{2} \circ f, J[f]\right]=\left(a a_{2}-b a_{1}\right) g_{z z}+\left(c a_{1}-a a_{3}\right) g_{z y} g_{z}+\left(a a_{3}-a_{1} c\right) g_{y} g_{z z}+ \\
\left(c a_{2}-b a_{3}\right) g_{x} g_{z z}+\left(b a_{3}-a_{2} c\right) g_{z} g_{z x} .
\end{gathered}
$$

Como $p_{2}$ é genérica então $\left(a a_{2}-b a_{1}\right) \neq 0$ e pela definição $\omega_{1}, \omega_{2}$ e $\omega_{3}$ são divisores inteiros do grau $d$.

Então a parte inicial do ideal é $\left(x, J[f],\left(a a_{2}-b a_{1}\right) g_{z z}\right)$ se $\omega_{1} \leq \omega_{2}$ ou $\left(x, J[f],\left(a a_{2}-b a_{1}\right) g_{z z}\right)$ se $\omega_{2} \leq \omega_{1}$. Estes ideais definem espaços que são I.C.I.S. e os graus respectivos de cada componente são $\omega_{1},\left(d-\omega_{3}\right),\left(d-3 \omega_{3}\right)$ e $\omega_{2},\left(d-\omega_{3}\right),\left(d-3 \omega_{3}\right)$.

O resultado segue agora do teorema 4.8 de Greuel-Hamm.

Demonstração do item 2. Usando a relação $\delta(f)-1=m_{0}(\Delta(f))$ em [4] teorema 3.3, facilmente é estabelecida a fórmula.

Demonstração do item 3. Aplicando os items 1. e 2. e o Teorema 4.4 para germes quase homogêneos e calculado o número de Milnor em termos dos pesos e graus para a hipersuperfície $\Sigma(f)$, obtemos a fórmula para a terceira multiplicidade polar $m_{2}(\Delta(f))$.

\subsection{Multiplicidades polares de $f\left(\Sigma^{1,1}(f)\right)$}

A seguir descreveremos as multiplicidades polares que surgem no conjunto $f\left(\Sigma^{1,1}(f)\right)$, que são duas, pois este é 1-dimensional.

Teorema 4.10 ([5], Teorema 3.2). Com as mesmas hipóteses do teorema 4.9, temos:

1. $m_{0}\left(f\left(\Sigma^{1,1}(f)\right)\right)=\frac{\left(d-\omega_{3}\right)\left(d-2 \omega_{3}\right)}{\max \left\{\omega_{1}, \omega_{2}\right\} \cdot \omega_{3}}$.

2. $m_{1}\left(f\left(\Sigma^{1,1}(f)\right)\right)=\sum_{j=1}^{2} \prod_{i=1}^{3}\left(\frac{d_{j}}{\omega_{i}}-1\right) \prod_{k=1, k \neq j}^{2}\left(\frac{d_{k}}{d_{j}-d_{k}}\right)+\frac{\prod_{s=1}^{2}\left(d-s \omega_{3}\right)\left(\omega_{1} \cdot \omega_{2}-\max \left\{\omega_{1}, \omega_{2}\right\}\left(d-3 \omega_{3}\right)\right)}{\omega_{1} \cdot \omega_{2} \cdot \omega_{3} \cdot \max \left\{\omega_{1}, \omega_{2}\right\}}-1$ 
Demonstração do item 1. Na demonstração do teorema 4.6 (ver [4] pág. 913) temos a seguinte igualdade

$$
m_{0}\left(f\left(\Sigma^{1,1}(f)\right)\right)=\operatorname{dim}_{\mathbb{C}} \frac{\mathscr{O}_{3}}{\left(I^{1,1}(f), p \circ f\right)}
$$

onde $I^{1,1}(f)$ é o ideal que define a curva cuspidal de $f, \Sigma^{1,1}(f)$ e $p$ é uma projeção genérica linear. Como $I^{1,1}(f)=\left(\frac{\partial g}{\partial z}, \frac{\partial^{2} g}{\partial z^{2}}\right)$, obtemos

$$
m_{0}\left(f\left(\Sigma^{1,1}(f)\right)\right)=\operatorname{dim}_{\mathbb{C}} \frac{\mathscr{O}_{3}}{\left(\frac{\partial g}{\partial z}, \frac{\partial^{2} g}{\partial z^{2}}, a x+b y+c g\right)}
$$

Então temos os seguintes casos:

Se $\omega_{1}<\omega_{2}$, o ideal $\left(\frac{\partial g}{\partial z}, \frac{\partial^{2} g}{\partial z^{2}}, a x+b y+c g\right)$ é semi quase homogêneo com parte inicial $\left(\frac{\partial g}{\partial z}, \frac{\partial^{2} g}{\partial z^{2}}, a x\right)$, para $a \neq 0$. Portanto

$$
m_{0}\left(f\left(\Sigma^{1,1}(f)\right)\right)=\frac{\left(d-\omega_{3}\right)\left(d-2 \omega_{3}\right)}{\omega_{2} \cdot \omega_{3}}
$$

Se $\omega_{2}<\omega_{1}$, o ideal $\left(\frac{\partial g}{\partial z}, \frac{\partial^{2} g}{\partial z^{2}}, a x+b y+c g\right)$ é semi quase homogêneo com parte inicial $\left(\frac{\partial g}{\partial z}, \frac{\partial^{2} g}{\partial z^{2}}, b y\right)$, $b \neq 0$. Portanto

$$
m_{0}\left(f\left(\Sigma^{1,1}(f)\right)\right)=\frac{\left(d-\omega_{3}\right)\left(d-2 \omega_{3}\right)}{\omega_{1} \cdot \omega_{3}}
$$

Juntando estas duas igualdades obtemos o teorema.

Demonstração do item 2. Para encontrar esta multiplicidade polar usaremos a relação do Teorema 4.6, isto, é,

$$
\left.\mu\left(\Sigma^{1,1}(f)\right)\right)+m_{0}\left(f\left(\Sigma^{1,1}(f)\right)\right)-1=m_{1}\left(f\left(\Sigma^{1,1}(f)\right)\right)+\# A_{3},
$$

pois segundo esta relação basta encontrar o número de Milnor da curva cúspidal $\Sigma^{1,1}(f)$ e o número de rabos de andorinhas de $f$.

Segundo os resultados de Greuel e Hamm em [14] e pelo capítulo 3, estes números são dados por:

$$
\mu\left(\Sigma^{1,1}(f)\right)=\sum_{j=1}^{2} \prod_{i=1}^{3}\left(\frac{d_{j}}{\omega_{i}}-1\right) \prod_{k=1, k \neq j}^{2}\left(\frac{d_{k}}{d_{j}-d_{k}}\right)
$$


e

$$
\# A_{3}=\frac{\omega_{3}^{2}}{\omega_{1} \cdot \omega_{2}} \prod_{j=1}^{3}\left(\frac{d}{\omega_{3}}-j\right)
$$

Portanto substituindo estes números na relação acima obtemos o resultado.

\subsection{Multiplicidades polares de $f\left(D_{1}^{2}(f / \Sigma(f))\right)$}

Nesta seção como nas anteriores não foi possível encontrar fórmulas independentementes para cada uma das multiplicidades polares. Isto, pelo fato de que a demonstração da relação entre os invariantes para $f\left(D_{1}^{2}(f / \Sigma(f))\right)$ envolve duas projeções diferentes. Mas encontramos uma fórmula para a diferença das multiplicidades polares. Inicialmente como corolário do teorema 4.5 , no caso de germes quase homogêneos de coposto 1 , obtemos uma relação entre as multiplicidades polares e os invariantes 0-estáveis.

Corolario 4.11 ([5]). Seja $f \in \mathscr{O}(3,3)$ germe quase homogêneo de coposto 1 . Então

$$
2 m_{0}\left(f\left(D_{1}^{2}(f / \Sigma(f))\right)\right)-2 m_{1}\left(f\left(D_{1}^{2}(f / \Sigma(f))\right)\right)=\# A_{1} A_{2}+5 \# A_{3}-\mu\left(D^{2}(f / \Sigma(f))\right)+1 .
$$

Demonstração: Para provar este corolário basta usar a proposição 4.7 e a fórmula dada no capítulo 3.

Com esta relação, as fórmulas do capítulo 3 e o resultado de K. Houston, Teorema 4.7, obtemos o seguinte teorema:

Teorema 4.12 ([5]). Com as hipóteses do teorema 4.9, temos

$$
2 m_{1}\left(f\left(D_{1}^{2}(f / \Sigma(f))\right)\right)-2 m_{0}\left(f\left(D_{1}^{2}(f / \Sigma(f))\right)\right)=\frac{\prod_{i=1}^{3}\left(d-i \omega_{3}\right)}{\omega_{1} \cdot \omega_{2} \cdot \omega_{3}}\left(2 d-\left(9 \omega_{3}+\omega_{1}+\omega_{2}\right)\right) .
$$




\section{Capítulo}

\section{5}

\section{Apêndice: Demonstração do Lema 2.1}

A demonstração deste resultado é feita em [3].

Observação 5.1. Considere $z=\left(z^{1}, \ldots, z^{1}, \ldots, z^{\ell}, \ldots, z^{\ell}\right)$ e $\Delta(\mathscr{P})$ definido como em 2.7. Então um ponto genérico de $\Delta(\mathscr{P})$ é da forma $\left(x, z^{1}, \ldots, z^{1}, \ldots, z^{\ell}, \ldots, z^{\ell}\right)$, com $z^{k}$ repetidos $r_{k}$-vezes $z^{i} \neq z^{k}, i \neq k$. Fixando $(x, \mathbf{z})=\left(x, z_{0}^{1}, \cdots, z_{r_{1}}^{1}, z_{0}^{2}, \cdots, z_{r_{2}}^{2}, \cdots, z_{0}^{\ell}, \cdots, z_{r_{\ell}}^{\ell}\right)$, Sejam

$$
\mathscr{E}_{0}^{S_{m+\ell}}\left(\mathbb{C}^{n-1+m+\ell}, \mathbb{C}^{m+\ell}\right)=\left\{\varphi:\left(\mathbb{C}^{n-1+m+\ell}, 0\right) \rightarrow \mathbb{C}^{m+\ell} \text { tal que } \varphi \text { é } S_{m+\ell}-\text { equivariante }\right\}
$$

e

$$
\mathscr{E}_{0}^{S_{m+\ell}}\left(\mathbb{C}^{n-1+m+\ell}\right)=\left\{\varphi:\left(\mathbb{C}^{n-1+m+\ell}, 0\right) \rightarrow \mathbb{C} \text { tal que } \varphi \text { é } S_{m+\ell}-\text { invariante }\right\}
$$

Seja $\mathscr{E}_{0}^{S_{m+\ell}}\left(\mathbb{C}^{n-1+m+\ell}, \mathbb{C}^{m+\ell}\right)$ o módulo finito sobre $\mathscr{E}_{0}^{S_{m+\ell}}\left(\mathbb{C}^{n-1+m+\ell}\right)$, gerado pelas aplicações

$$
\boldsymbol{\varphi}_{i}(x, \mathbf{z})=\left(\left(z_{0}^{1}\right)^{i}, \ldots,\left(z_{r_{1}}^{1}\right)^{i}, \ldots,\left(z_{0}^{\ell}\right)^{i}, \ldots,\left(z_{r_{\ell}}^{\ell}\right)^{i}\right)
$$

para $0 \leq i \leq m+\ell-1$

Lema 5.2 ([3], Lema 2.4). Para qualquer função $q \in \mathscr{O}_{n}, q=q(x, z)$, seja $\mathscr{J}_{m+\ell}(q)$ o ideal gerado pelos coeficientes $\alpha_{1}, \ldots, \alpha_{m+\ell-1}$ na equação

$$
\left(q\left(x, z_{0}^{1}\right),, \ldots, q\left(z, z_{0}^{m+\ell}\right)\right)=\sum_{i=0}^{m+\ell-1} \alpha_{i}\left(x, z_{0}^{1}, \ldots, z_{0}^{m+\ell}\right) \varphi_{i}\left(x,\left(z_{0}^{1}\right)^{i}, \ldots,\left(z_{0}^{m+\ell}\right)^{i}\right)
$$

com $\varphi_{i}$ sendo como na observação anterior e $\alpha_{i} \in \mathscr{E}_{0}^{S_{m+\ell}}\left(\mathbb{C}^{n-1+m+\ell}\right)$. Então,

$$
\begin{gathered}
\mathscr{J}_{m+\ell}(q)+\left(z_{0}^{1}-z_{1}^{1}, \ldots, z_{0}^{1}-z_{r_{1}}^{1}, z_{0}^{1}-z_{0}^{2}, \ldots, z_{0}^{1}-z_{r_{2}}^{2}, \ldots, z_{0}^{1}-z_{0}^{\ell}, \ldots, z_{0}^{1}-z_{r_{\ell}}^{\ell}\right)= \\
\left(\frac{\partial q}{\partial z}, \ldots, \frac{\partial^{m+\ell-1} q}{\partial z^{m+\ell-1}}\right)+\left(z_{0}^{1}-z_{1}^{1}, \ldots, z_{0}^{1}-z_{r_{1}}^{1}, z_{0}^{1}-z_{0}^{2}, \ldots, z_{0}^{1}-z_{r_{2}}^{2}, \ldots, z_{0}^{1}-z_{0}^{\ell}, \ldots, z_{0}^{1}-z_{r_{\ell}}^{\ell}\right)
\end{gathered}
$$


Demonstração: Seja $\mathscr{P}=\left(r_{1}, \ldots, r_{\ell}\right)$ partição de $m$. Como $\left(z_{0}^{1}-z_{1}^{1}, \ldots, z_{0}^{1}-z_{r_{\ell}}^{\ell}\right)$ é o ideal de funções que se anula sobre a diagonal $\Delta_{(m+\ell)}=\left\{\left(x, z_{0}^{1}, \ldots, z_{r_{\ell}}^{\ell} \mid z_{i}^{k}=z_{j}^{k}, \forall i, j, k=1, \ldots, \ell\right\}\right.$, é necessário somente mostrar que o ideal em $\mathscr{O}_{\Delta_{(m+\ell)}}$ gerado pelas restrições de $\alpha_{i}$, com $1 \leq i \leq$ $m+\ell-1$, coincide com o ideal gerado pelas primeiras $m+\ell-1$ derivadas parciais de $q$ com respeito a $z$.

Escreva $z_{0}^{1}=z, z_{1}^{1}=z+\varepsilon, \ldots, z_{r_{\ell}}^{\ell}=z+(m+\ell-1) \varepsilon$, e seja $q_{s}=s ! \frac{\partial^{s} q}{\partial z^{s}}$. Expandindo o lado esquerdo da igualdade 5.2, usando o teorema de Taylor e usando o vetor coluna ao invés do vetor linha na notação, obtemos:

$$
\begin{gathered}
{\left[\begin{array}{c}
q \\
q+\varepsilon q_{1}+\ldots+\varepsilon^{m+\ell-1} q_{m+\ell-1}+R_{1} \\
\vdots \\
q+(m+\ell-1) \varepsilon q_{1}+\ldots+((m+\ell-1) \varepsilon)^{m+\ell-1} q_{m+\ell-1}+R_{1}
\end{array}\right]=\alpha_{0}\left[\begin{array}{c}
1 \\
1 \\
\vdots \\
1
\end{array}\right]+} \\
+\alpha_{1}\left[\begin{array}{c}
z \\
z+\varepsilon \\
\vdots \\
z+(m+\ell-1) \varepsilon
\end{array}\right]+\ldots+\alpha_{m+\ell-1}\left[\begin{array}{c}
z^{m+\ell-1} \\
(z+\varepsilon)^{m+\ell-1} \\
\vdots \\
(z+(m+\ell-1) \varepsilon)^{m+\ell-1}
\end{array}\right]
\end{gathered}
$$

Cada termo $R_{i}$ satisfaz $\lim _{\varepsilon \rightarrow 0} \frac{R_{i}}{\varepsilon^{m+\ell-1}}=0$.

Seja $M$ a matriz

$$
\left[\begin{array}{cccc}
1 & 0 & \cdots & 0 \\
1 & \varepsilon & \cdots & \varepsilon^{m+\ell-1} \\
\vdots & \vdots & & \vdots \\
1 & (m+\ell-1) \varepsilon & \cdots & ((m+\ell-1) \varepsilon)^{m+\ell-1}
\end{array}\right]
$$

Então $M$ divide a matriz dos coeficientes de $\alpha_{i}$ do lado direito de 5.3, e realmente quando $\varepsilon \neq 0$ (e portanto $M$ é invertível) podemos escrever: 


$$
\begin{gathered}
M\left\{\left[\begin{array}{c}
q \\
q_{1} \\
\vdots \\
q_{m+\ell-1}
\end{array}\right]+M^{-1}\left[\begin{array}{c}
0 \\
R_{1} \\
\vdots \\
R_{m+\ell-1}
\end{array}\right]\right\}= \\
M\left[\begin{array}{ccccc}
1 & z & z^{2} & \cdots & z^{m+\ell-1} \\
0 & 1 & 2 z & \cdots & (m+\ell-1) z^{m+\ell-2} \\
& 0 & 1 & & \\
\vdots & \vdots & \vdots & & \vdots \\
0 & 0 & 0 & \cdots & 1
\end{array}\right]\left[\begin{array}{c}
\alpha_{0} \\
\alpha_{1} \\
\vdots \\
\alpha_{m+\ell-1}
\end{array}\right]
\end{gathered}
$$

Afirmamos que $\lim _{\mathcal{E} \rightarrow 0} M^{-1} R=0$ (onde $R$ é o vetor coluna $\left(0, R_{1}, \ldots, R_{m+\ell-1}\right)^{t}$ ).

Isto é mostrado contando as potências de $\varepsilon$ : escrevendo $M^{-1}=(\operatorname{det} M)^{-1} a d j(M)$, e notando que $\operatorname{det} M$ é um múltiplo de $\varepsilon^{\frac{1}{2}(m+\ell)(m+\ell-1)}$, enquanto cada entrada em $\operatorname{adj}(M)$ tem ordem em $\varepsilon$ em ao menos $\frac{1}{2}(m+\ell-2)(m+\ell-1)$, vemos que cada entrada em $M^{-1}$ tem ordem ao menos $(m+\ell-1)$, como $R_{i}=0\left(\varepsilon^{m+\ell-1}\right)$ segue a afirmação.

De 5.4, a continuidade de $q_{i}$ e $\alpha_{i}$, e o fato que $\lim _{\varepsilon \rightarrow 0} M^{-1} R=0$, deduzimos que, quando $\varepsilon=0$,

$$
\left(q, q_{1}, \ldots, q_{m+\ell-1}\right)^{t}=P\left(\alpha_{0}, \ldots, \alpha_{m+\ell-1}\right)^{t}
$$

onde $P$ é a matriz de Pascal do lado direito de 5.4. Tirando a primeira linha e coluna de $P$, temos

$$
\left(q_{1}, \ldots, q_{m+\ell-1}\right)^{t}=\bar{P}\left(\alpha_{1}, \ldots, \alpha_{m+\ell-1}\right)^{t}
$$

e portanto, $\bar{P}$ é invertível e o lema está provado.

Finalmente temos condições de provar o lema 2.1. 
Lema 2.1[[3], Lema 2.7] Em um ponto genérico de $\Delta(\mathscr{P})$ temos,

$$
\begin{aligned}
\mathscr{J}_{\Delta}^{\ell}(f, \mathscr{P})= & \left\langle\frac{\partial g}{\partial z}\left(x, z^{1}\right), \cdots, \frac{\partial^{r_{1}} g}{\partial z^{r_{1}}}\left(x, z^{1}\right), \cdots, \frac{\partial g}{\partial z}\left(x, z^{\ell}\right), \cdots, \frac{\partial^{r} \ell g}{\partial z^{r_{\ell}}}\left(x, z^{\ell}\right)\right\rangle \\
& +\left\langle g\left(x, z^{i}\right)-g\left(x, z^{1}\right) ; 2 \leq i \leq \ell\right\rangle+\mathscr{I}_{\Delta(\mathscr{P})} .
\end{aligned}
$$

Demonstração: $\mathrm{O}$ caso $\mathscr{P}=(m)$ já está provado pelo lema 5.2.

Como $\mathscr{I}_{\Delta}(\mathscr{P})$ está contido em ambos os ideais cuja igualdade queremos provar, precisamos mostrar somente que a restrição à $\Delta(\mathscr{P})$ dos geradores de $\mathscr{J}^{\ell}(f, \mathscr{P})$ gera o mesmo ideal em $\mathscr{O}_{\Delta(\mathscr{P}),(x, z)}$ com os elementos listados no lado direito da igualdade do lema.

Seja $G=S_{r_{1}} \times \ldots \times S_{r_{\ell}}, G$ age sobre $\mathbb{C}^{n-1+m+\ell}$ permutando suas últimas $(m+\ell)$-coordenadas e sobre $\mathbb{C}^{m+\ell}$ permutando suas coordenadas, cada $S_{r_{i}}, i=1, \ldots, \ell$ permutas as coordenadas $z_{0}^{i}, \ldots, z_{r_{i}}^{i}$.

Por [[17], pág 106], vê-se que os $(m+\ell)$-vetores $\varphi_{k}^{s}$, com $\left(1 \leq s \leq \ell, 0 \leq k \leq r_{s}\right)$, e com $\left(z_{a}^{s}\right)^{k}, 0 \leq a \leq r_{s}$ no $\rho(a, s)$-ésimo lugar e zero nas outras posições, geram o módulo, onde $\rho(a, s)=a+s+r_{1}+\cdots+r_{s-1}$.

Sejam $N(G)$ a matriz com colunas $\varphi_{0}^{1}, \ldots, \varphi_{r_{1}}^{1}, \ldots, \varphi_{0}^{\ell}, \ldots, \varphi_{r_{\ell}}^{\ell}$ e $N\left(S_{m+\ell}\right)$ a matriz cujas colunas são os geradores $\varphi_{0}, \ldots, \varphi_{m+\ell-1}$ de $\mathscr{E}_{0}^{S_{m+\ell}}\left(\mathbb{C}^{n-1+m+\ell}, \mathbb{C}^{m+\ell}\right)$ sobre $\mathscr{E}_{0} S_{m+\ell}\left(\mathbb{C}^{n-1+m+\ell}\right)$ como na observação 5.1. Então como $\mathscr{E}_{0}^{S_{m+\ell}}\left(\mathbb{C}^{n-1+m+\ell}, \mathbb{C}^{m+\ell}\right) \subset \mathscr{E}_{0} G\left(\mathbb{C}^{n-1+m+\ell}, \mathbb{C}^{m+\ell}\right), N(G)$ divide $N\left(S_{m+\ell}\right)$, daí,

$$
N\left(S_{m+\ell}\right)=N(G) Q
$$

$N\left(S_{m+\ell}\right)$ e $N(G)$ são invertíveis e $\operatorname{det}(Q)=\operatorname{det} N(G)^{-1} \operatorname{det} N\left(S_{m+\ell}\right)$ não se anula em um ponto genérico $(x, z)$ de $\Delta(\mathscr{P})$.

Seja $L:\left(\mathbb{C}^{n-1+m+\ell}, 0\right) \rightarrow\left(\mathbb{C}^{m+\ell}, 0\right)$ a aplicação dada por:

$$
L(x, \mathbf{z})=\left(g\left(x, z_{0}^{1}\right), \ldots, g\left(x, z_{r_{1}}^{1}\right), \ldots, g\left(x, z_{0}^{\ell}\right), \ldots, g\left(x, z_{r_{\ell}}^{\ell}\right)\right)
$$

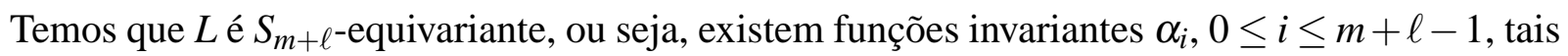
que $L=\sum_{i=0}^{m+\ell-1} \alpha_{i} \varphi_{i}$, então, $L \in \mathscr{E}_{0}^{S_{m+\ell}}\left(\mathbb{C}^{n-1+m+\ell}, \mathbb{C}^{m+\ell}\right) \subset \mathscr{E}_{0} G\left(\mathbb{C}^{n-1+m+\ell}, \mathbb{C}^{m+\ell}\right)$, logo podemos escrever 


$$
N(G) \beta=N\left(S_{m+\ell}\right) \alpha=N(G) Q \alpha
$$

para algum vetor coluna $\beta=\left(\beta_{0}^{1}, \ldots, \beta_{r_{1}}^{1}, \ldots, \beta_{0}^{\ell}, \ldots, \beta_{r_{\ell}}^{\ell}\right)^{t}$ e $\alpha=\left(\alpha_{0}, \ldots, \alpha_{m+\ell-1}\right)^{t}$, com $\alpha_{i}=$ $h_{i}, h_{i} \in \mathscr{J}^{\ell}(f, \mathscr{P}), 1 \leq i \leq m+\ell-1$. Como $N(G)$ é invertível sobre um subconjunto aberto denso de qualquer vizinhança de $(x, z)$, podemos cancelar $N(G)$ em 5.6 e obter,

$$
\beta=Q \alpha
$$

Além de obter uma equação relacionando $\beta_{a}^{s}$ com os geradores $\alpha_{0}, \ldots, \alpha_{m+\ell-1}$ de $\mathscr{J}^{\ell}(f, \mathscr{P})$, reduzimos a primeira coluna de $Q$ à 1 na primeiro casa, seguidos de zeros no resto, subtraindo a primeira linha das $\left(\left(r_{1}+1\right)+1\right)$-ésimas, $\left.\left.\ldots,\left(\left(r_{1}+1\right)+1\right)+\left(r_{2}+1\right)+1\right)+\cdots+\left(r_{s-1}+1\right)+1\right)$ ésimas linhas.

Assim, a equação 5.7 se escreve como:

$$
\left[\begin{array}{c}
\beta_{0}^{1} \\
\vdots \\
\beta_{r_{1}}^{1} \\
\beta_{0}^{2}-\beta_{0}^{1} \\
\vdots \\
\beta_{r_{2}}^{2} \\
\beta_{0}^{\ell}-\beta_{0}^{1} \\
\vdots \\
\beta_{r_{\ell}}^{\ell}
\end{array}\right]=\left[\begin{array}{c}
1 \\
\hline 0 \\
\\
\\
\end{array}\right]
$$

Pelo Lema 5.2, quando restringimos a diagonal $\Delta(\mathscr{P})$, temos que o ideal em $\mathscr{O}_{\Delta(\mathscr{P}),(x, z)}$ gerado por $\beta_{1}^{s}, \cdots, \beta_{r_{s}}^{s}$ é igual ao ideal $J_{s}$ gerado por $\frac{\partial g}{\partial z}\left(x, z^{s}\right), \cdots, \frac{\partial^{r_{s}} g}{\partial z^{r_{s}}}\left(x, z^{s}\right)$. Além disso, pela demonstração do Lema 5.2, vemos que $\beta_{0}^{s}$ módulo o ideal $J_{s}$ é apenas $g\left(x, z^{s}\right)$, isto é, $\beta_{0}^{s}=$ $g\left(x, z^{s}\right)+\theta_{s}, \theta_{s} \in J_{s}, \operatorname{logo}, \beta_{0}^{s}-\beta_{0}^{1}=g\left(x, z^{s}\right)-g\left(x, z^{1}\right)+\left(\theta_{s}-\theta_{1}\right), \theta_{s}-\theta_{1} \in J_{s}+J_{1}$, então, $\beta_{0}^{s}-\beta_{0}^{1}$ módulo o ideal $\left(J_{s}+J_{1}\right)$ é $g\left(x, z^{s}\right)-g\left(x, z^{1}\right), 2 \leq s \leq \ell, \operatorname{como} \operatorname{det} Q=\operatorname{det} Q^{\prime}, Q^{\prime}$ é invertível em $(x, z)$ e portanto o lema está provado. 
Exemplo 5.3. Veremos agora um exemplo que verifica a igualdade 5.5

Consideremos $m=n=2$ e a partição $\mathscr{P}=(1,1)$, assim temos $r_{1}=r_{2}=1, \ell=2$. Sabemos que os $(m+\ell)$-vetores $\varphi_{k}^{s},\left(1 \leq s \leq \ell, 0 \leq k \leq r_{s}\right)$, com $\left(z_{a}^{s}\right)^{k}$, no $\rho(a, s)$-ésimo lugar e zero nas outras posições, geram o módulo, onde $0 \leq a \leq r_{s}$ e $\rho(a, s)=a+s+r_{1}+\cdots+r_{s-1}$.

$N(G)$ é a matriz com colunas $\varphi_{0}^{1}, \ldots, \varphi_{r_{1}}^{1}, \ldots, \varphi_{0}^{\ell}, \ldots, \varphi_{r_{\ell}}^{\ell}$

$N\left(S_{m+\ell}\right)$ é a matriz cujas colunas são os geradores $\varphi_{0}, \ldots, \varphi_{m+\ell-1}$ do módulo, onde $\varphi_{i}$ é a igualdade 5.1 na observação 5.1

Como $1 \leq s \leq \ell=2$ e $0 \leq k \leq r_{s}$, então para

$$
\begin{aligned}
& s=1 \rightarrow k=0,1 \rightarrow \varphi_{k}^{s}=\left(\varphi_{0}^{1}, \varphi_{1}^{1}\right) \\
& s=2 \rightarrow k=0,1 \rightarrow \varphi_{k}^{s}=\left(\varphi_{0}^{2}, \varphi_{1}^{2}\right)
\end{aligned}
$$

Assim temos os seguintes vetores $\varphi_{k}^{s}$

$$
\left\{\begin{array}{l}
\left.\varphi_{0}^{1}=\left(\left(z_{0}^{1}\right)^{0},\left(z_{1}^{1}\right)^{0}, 0,0\right)\right)=(1,1,0,0) \\
\left.\varphi_{1}^{1}=\left(\left(z_{0}^{1}\right)^{1},\left(z_{1}^{1}\right)^{1}, 0,0\right)\right)=\left(z_{0}^{1}, z_{1}^{1}, 0,0\right) \\
\left.\varphi_{0}^{2}=\left(0,0,\left(z_{0}^{2}\right)^{0},\left(z_{1}^{2}\right)^{0}\right)\right)=(0,0,1,1) \\
\left.\varphi_{1}^{2}=\left(0,0,\left(z_{0}^{2}\right)^{1},\left(z_{1}^{2}\right)^{1}\right)\right)=\left(0,0, z_{0}^{2}, z_{1}^{2}\right)
\end{array}\right.
$$

Daí temos que

$$
N(G)=\left[\begin{array}{cccc}
1 & z_{0}^{1} & 0 & 0 \\
1 & z_{1}^{1} & 0 & 0 \\
0 & 0 & 1 & z_{0}^{2} \\
0 & 0 & 1 & z_{1}^{2}
\end{array}\right]
$$

e

$$
N\left(S_{m+\ell}\right)=\left[\begin{array}{cccc}
1 & z_{0}^{1} & \left(z_{0}^{1}\right)^{2} & \left(z_{0}^{1}\right)^{3} \\
1 & z_{1}^{1} & \left(z_{1}^{1}\right)^{2} & \left(z_{1}^{1}\right)^{3} \\
1 & z_{0}^{2} & \left(z_{0}^{2}\right)^{2} & \left(z_{0}^{2}\right)^{3} \\
1 & z_{1}^{2} & \left(z_{1}^{2}\right)^{2} & \left(z_{1}^{2}\right)^{3}
\end{array}\right]
$$


Deste modo a matriz $Q$ que satisfaz a igualdade 5.5 é a seguinte:

$$
Q=\left[\begin{array}{cccc}
1 & 0 & -z_{0}^{1} z_{1}^{1} & -z_{1}^{1}\left(z_{0}^{1}\right)^{2}-z_{0}^{1}\left(z_{1}^{1}\right)^{2} \\
0 & 1 & z_{0}^{1}+z_{1}^{1} & \left(z_{0}^{1}\right)^{2}+z_{0}^{1} z_{1}^{1}+\left(z_{1}^{1}\right)^{2} \\
1 & 0 & -z_{0}^{2} z_{1}^{2} & -\left(z_{0}^{2}\right)^{2} z_{1}^{2}-z_{0}^{2}\left(z_{1}^{2}\right)^{2} \\
0 & 1 & z_{0}^{2}+z_{1}^{2} & \left(z_{0}^{2}\right)^{2}+z_{0}^{2} z_{1}^{2}+\left(z_{1}^{2}\right)^{2}
\end{array}\right]
$$




\section{Referências Bibliográficas}

[1] Jorge Perez, V. H. and Levcovitz, D. and Saia, M. J., Invariants, equisingularity and Euler obstruction of map germs from $\mathbb{C}^{n}$ to $\mathbb{C}^{n}$, A ser publicado no Crelle's Journal em 2005.

[2] Marar, W. L., Montaldi, J. A. and Ruas, M. A. S., Multiplicities of zero-schems in quasihomogeneous corank-1 singularities, Singularity Theory (Liverpool, 1996), 353-367, London Math. Soc. Lecture Note ser., 263, Camb. Univ. Press, Cambridge, 1999.

[3] Marar, W. L. and Mond, D., Multiple point schemes for corank 1 maps, J. London Math, Soc. $39,1989,553-567$.

[4] Jorge Pérez, V. H., Polar multiplicities and equisingularity of map germs from $\mathbb{C}^{3}$ to $\mathbb{C}^{3}$, Houston Journal of Mathematics. 29 n: 04, 2003, 901-923,

[5] Jorge Pérez, V. H., Weighted homogeneous map germs of corank one from $\mathbb{C}^{3}$ to $\mathbb{C}^{3}$ and polar multiplicities, Universidad Católica del Norte. Vol. 21, nº 3, 2002, 245-259,

[6] Gunning, R. T., Lectures on complex analytic varieties, Princeton, New Jersey, 1970.

[7] Gaffney, T. and Mond, D., Weighted homogeneous maps from the plane to the plane, Math. Proc. Camb. Phil, Soc. 109, 1991, 451-470.

[8] Looijenga, E. J. N., Isolated singular points on complete intersections, London Mathematical Soc. Lecture Note Series. 77, 1984.

[9] Mond, D. and Pellikaan, R., Fitting ideals and multiple points of analitics maps, Springer L.N.M 1414, 1989, 553-567.

[10] Gaffney, T., Polar Multiplicities and Equisingularity of Map Germs, Topology,1993, vol.32, No.1, 185-223. 
[11] Gaffney, T., Plane Sections, $W_{f}$ and $A_{f}$, in Real Complex Singularities, Bruce, J. W. and Tari, F.; Research Notes in Mathematics 412, Chapman and Hall, 2000.

[12] Gaffney, T. and Massey, D., Trends in Equisingularity Theory in Singularity Theory, Bruce, J.W. and Mond. D., Cambridge University Press, 1999.

[13] Tessier, B., Variétés Polaires 2: Multiplicités Polaires, Sections Planes, et Conditions de Whitney, Actes de la conference de géometrie algébrique la Rábida, Springer Lecture Notes, 961, 1981, 314-491.

[14] Greuel, G. M. and Hamm, H. A., Invarianten quasihomogener vollstädiger Durchschnitte, Invent. Math. 49, 1978, 67-86.

[15] Houston, K., Generalised image and discriminant Milnor numbers for corank 1 mappings. July-1998. Preprint.

[16] Wall, C.T.C., Lectures on $C^{\infty}$-Stability and Classifications, Springer Lecture Notes in Mathematics, No.192, 1971, 178-206.

[17] Poénaru, V., Singularities $C^{\infty}$ en présence de simétrie, Lecture Notes in Mathematics 510, Springer, Berlin, 1976.

[18] Ruas, M. A. S., On the equisingularity of families of corank 1 generic germs, Contemporacy Mathematics, 161, 1994, 113-121. 